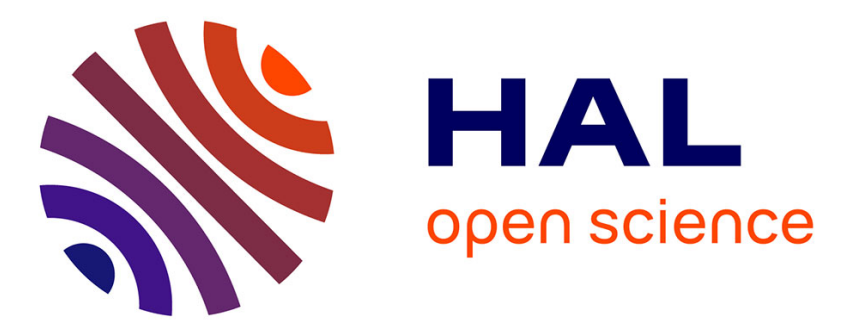

\title{
Boundary integral formulation and two-dimensional fundamental solutions for dynamic behavior analysis of unsaturated soils
}

\author{
Pooneh Maghoul, Behrouz Gatmiri, Denis Duhamel
}

\section{To cite this version:}

Pooneh Maghoul, Behrouz Gatmiri, Denis Duhamel. Boundary integral formulation and twodimensional fundamental solutions for dynamic behavior analysis of unsaturated soils. Soil Dynamics and Earthquake Engineering, 2011, 31 (11), pp.1480-1495. 10.1016/j.soildyn.2011.05.016 . hal-00668419

\section{HAL Id: hal-00668419 \\ https://hal.science/hal-00668419}

Submitted on 9 Feb 2012

HAL is a multi-disciplinary open access archive for the deposit and dissemination of scientific research documents, whether they are published or not. The documents may come from teaching and research institutions in France or abroad, or from public or private research centers.
L'archive ouverte pluridisciplinaire HAL, est destinée au dépôt et à la diffusion de documents scientifiques de niveau recherche, publiés ou non, émanant des établissements d'enseignement et de recherche français ou étrangers, des laboratoires publics ou privés. 


\title{
Boundary Integral Formulation and Two-Dimensional Fundamental Solution for Dynamic Behaviour Analysis of Unsaturated Soils
}

\author{
Pooneh Maghoul $^{\mathrm{a}}$, Behrouz Gatmiri ${ }^{\mathrm{a}, \mathrm{b}, *}$, Denis Duhamel $^{\mathrm{a}}$ \\ ${ }^{a}$ Université Paris-Est, UR Navier, Ecole des Ponts, 6 et 8 Avenue Blaise Pascal, Cité \\ Descartes, Champs sur Marne, 77455 Marne la Vallée, Cedex 2, France \\ ${ }^{b}$ Departments of Civil Engineering, University of Tehran, P.O. Box 11365-4563, Tehran, \\ Iran
}

\begin{abstract}
.
In this paper the coupled equations governing the dynamic behaviour of unsaturated soils are derived based on the poromechanics theory in the frame of the suction-based mathematical model presented by Gatmiri (1997) and Gatmiri et al. (1998). In this formulation, the solid skeleton displacements, water pressure and air pressure are presumed as independent variables. The Boundary Integral formulations as well as fundamental solutions for such dynamic $u-p_{w}-p_{a}$ theory are presented in this paper for the first time. The boundary integral equations are derived via the use of weighted residuals method in a way that permits an easy discretization and implementation in a Boundary Element code. Also, the associated two dimensional (2D) fundamental solutions for such deformable porous medium with linear elastic behavior are derived in Laplace transform domain using the method of Hörmander. Finally, some numerical results are presented to show the accuracy of the proposed solutions. The derived results are verified analytically by comparison with the previously introduced corresponding fundamental solutions in elastodynamic limiting case.
\end{abstract}

Keywords: boundary element method; boundary integral equation; fundamental solution; singular behaviour; unsaturated soil; multiphase porous medium; dynamic behaviour

\section{Introduction}


Unsaturated soils are encountered near the earth surface where most of engineering structures are ultimately supported. Even though geotechnical engineering projects encounter saturated, dry and unsaturated soils, most of the past studies have been done only on saturated and dry soils. Saturated and dry soils can become unsaturated due to seasonal variations.

The dynamic behavior of the saturated soils has been extensively investigated $[3,4,53,41,42$ among others]. In the current state of the knowledge, it could be claimed that behaviour of the saturated porous media has been well understood. In contrast, the study of the dynamic behavior of the unsaturated porous media is a relatively new area in the field of geotechnical earthquake engineering. Accurate measurement of various quantities such as dynamic water and air pressures, and degree of saturation in partially saturated soils is a difficult task during dynamic loadings [43].

Wave propagation in unsaturated soils in arid areas and the dynamic response of such media are of great interest in geophysics, soil and rock mechanics, and many earthquake engineering problems. But, in geomechanics, the behaviour of many media including more than two phases is not consistent with the principles and concepts of classical saturated soil mechanics. Thus, the prediction and simulation of unsaturated soil behaviour are of great importance in making critical decisions that affect many facets of engineering design and construction.

An unsaturated porous medium can be represented as a three-phase (gas, liquid, and solid), or three-component (water, dry air, and solid) system in which two phases can be classified as fluids (i.e. liquid and gas). The liquid phase is considered to be pure water containing dissolved air and the gas phase is assumed to be a binary mixture of water vapor and 'dry' air in a non-isothermal case. The air in an unsaturated soil may be in an occluded form when the degree of saturation is relatively high. At a lower degree of saturation, the gas phase is continuous.

In order to model unsaturated soil behaviour, first the governing partial differential equations should be derived and solved. Because of the complexity of the governing partial differential equations, with the exception of some simple cases, their closed-form solutions are not available. Therefore the numerical methods, such the Finite Element Method (FEM) and the Boundary Element Method (BEM), should be used for such partial differential equations.

The FEM has proven to be very effective in solving problems with bounded domains, particularly when inhomogenities and non-linear effects should be treated. Then, regarding its vast ability in geomechanics, it has been used in many codes. One of these codes that has been developed to model the different aspects of unsaturated soils is $(\theta-\mathrm{STOCK})$ program which is written by Gatmiri (1997) [14]. This code has been validated by several applications. For the 
sake of brevity of the text the literature review has been omitted. An exhaustive literature review has been given in [26, 22, 23, 28].

For domains of infinite extensions however, standard finite elements discretization leads to wave reflections at the edges of the FE mesh, which can be only partly eliminated for some cases, by using so-called transmitting, silent and non-reflecting viscous boundaries [37, 52]. Other solutions, such as consistent infinitesimal FE cell methods, because of their important disadvantage of being formulated in transformed spaces, seem cannot be used in non-linear dynamic analysis [31].

The BEM, on the other hand, is a very effective numerical tool for dynamic analysis of linear elastic bounded and unbounded media. The method is very attractive for wave propagation problems, because the discretization is done only on the boundary, yielding smaller meshes and systems of equations. Another advantage is that this method represents efficiently the outgoing waves through infinite domains, which is very useful when dealing with scattered waves by topographical structures. When this method is applied to problems with semiinfinite domains, there is no need to model the far field. In this method, during the formulation of boundary integral equations, the fundamental solutions for the governing partial differential equations should be first derived. Indeed, attempting to solve numerically the boundary value problems for unsaturated soils using BEM leads one to search for the associated fundamental solutions.

To the best of the authors' knowledge, no fundamental solution exists in the published literature for the dynamic modeling of unsaturated soils so far, hence the development of a BEM model for dynamic behaviour of unsaturated soil is not yet possible.

In the saturated media, it seems that the first attempt to obtain fundamental solutions for dynamic poroelasticity was presented by Burridge and Vargas (1979) [6] for the $u_{i}^{s}-u_{i}^{w}$ formulation who gave a general solution procedure similar to that of Deresiewicz (1960) [11]. As inhomogeneity they chose only a point force in the solid which is not sufficient for the usage of such a fundamental solution in a BE formulation. Later, Norris (1985) [40] derived time harmonic Green functions for the same formulation for a point force in the solid as well as a point force in the fluid. He also obtained explicit asymptotic approximations for far-field displacements, as well as those for low and high frequency responses. Afterwards, Kaynia and Banerjee (1993) [33] used a solution scheme similar to that of Norris (1985) [40] and derived the fundamental solution in the Laplace transform domain as well as transient short-time solution. The Burridge and Vargas solution was obtained for three forces, while those of 
Norris, and Kaynia and Banerjee have used six variables (displacements of the solid skeleton and average displacements of the fluid), both of which seem not to be adequate for application. The first approach does not have enough variables and the second one has too many. Manolis and Beskos (1989) [39] have pointed out the analogy between poroelasticity and thermoelasticity. However, this analogy is only possible for the $u_{i}^{s}-p$ formulation. It is also shown by Bonnet (1987) [5] when he presented the fundamental solution for the $u_{i}^{s}-p$ formulation in frequency domain. Further, he concluded that the $u_{i}^{s}-p$ formulation is sufficient and the $u_{i}^{s}-u_{i}^{w}$ formulation is overdetermined. Dominguez (1991) [12] presented the basic formulation of the frequency domain integral formulation for dynamic poroelasticity in terms of solid displacements and fluid stress. In another paper, Dominguez (1992) [13] presented a boundary element approach for dynamic poroelastic problems in frequency domain. In this paper Dominguez used the equivalence between poroelasticity and thermoelasticity for obtaining the fundamental solution. Also Weibe and Antes (1991) [51] seem to be the first who obtained a time domain two-dimensional (2D) fundamental solution for the Biot type dynamic poroelasticity for the $u_{i}^{s}-u_{i}^{w}$ formulation by neglecting the viscous coupling and without numerical evaluation of the kernel functions. Without this restriction Chen (1994 a, b) [8, 9] proposed another approximate transient 2D and 3D fundamental solutions for the special case of short time as well as the general case for the $u_{i}^{s}-p$ formulation, which were too complicated to be applied in BE algorithms. Later Gatmiri and Kamalian (2002) [16] showed that Chen's approximation could not be used in the simplified case of $u_{i}^{s}-p$ formulation. They derived other approximate transient 2D fundamental solutions for the $u_{i}^{s}-p$ formulation. Later Gatmiri and Nguyen (2005) [21] proposed closedform transient 2D fundamental solutions for the $u_{i}^{s}-p$ formulation of saturated porous media consisting of incompressible constituents. They have shown that their solution is a very accurate solution especially in long time. More recently Schanz and Pryl (2004) [48] have derived dynamic fundamental solutions for deformable solid skeleton with compressible and incompressible fluid in Laplace transform domain. By comparison of the two sets of the derived fundamental solution, they have concluded that an incompressible model can only be used in wave propagation problems if the short time behaviour is not considered and also if the ratios of the compression moduli are very insignificant. Also, the Green functions for a continuously non-homogeneous saturated media obeying Biot's dynamic poroelastic theory, 
have been derived by Seyrafian et al. (2006) [45]. Just recently, Gatmiri and Eslami (2007) [25] presented an analytical solution for the evaluation of scattering of waves by a circular cavity in infinite isotropic elastic porous media.

For unsaturated soils, Gatmiri and Jabbari (2004a, b, 2005a, b) [17, 18, 19, 20] have derived the first fundamental solution for the nonlinear governing differential equations for static and quasistatic poroelastic media for both two and three-dimensional problems. The corresponding thermo-poro-mechanic fundamental solutions for static and quasi-static problems are, respectively, derived by Jabbari and Gatmiri (2007) [30] (for both two and three dimensional problems) and Gatmiri et al. (2009) [24] (for two-dimensional problems) and Maghoul et al. (2009) [38] (for three-dimensional problems).

This paper in consecrated to obtain the boundary integral equation and $2 \mathrm{D}$ fundamental solution for unsaturated soils under dynamic loadings in order to be able to model the wave propagation phenomena in these media by BEM.

In this paper first, the set of fully coupled governing differential equations of a porous medium saturated by two compressible fluids (water and air) subjected to dynamic loadings is obtained. These phenomenal formulations are presented based on the experimental observations and with respect to the poromechanics theory in the frame of the suction-based mathematical model presented by Gatmiri (1997) [14] and Gatmiri et al. (1998) [15].

In this model, the effect of deformations on the suction distribution in soil skeleton and the inverse effect are included in the formulation via a suction-dependent formulation of state surfaces of void ratio and degree of saturation. The linear constitutive law is assumed. The mechanical and hydraulic properties of porous media are assumed to be suction dependent. In this formulation, the solid skeleton displacements, water pressure and air pressure are presumed as independent variables.

Secondly, the Boundary Integral Equation (BIE) is developed directly from those equations via the use of weighted residuals method for the first time in a way that permits an easy discretization and implementation in a numerical code.

The associated fundamental solution in Laplace transformed domain is presented by the use of the method of Hörmander (1963) [29] or Kupradze (1979) [34] for 2D $u_{i}-p_{w}-p_{a}$ formulation of unsaturated porous media. As these solutions are the basis of BE formulation their singular behavior is also discussed.

In this case that fundamental solution is known only in the frequency domain and it is impossible to obtain the time-dependent fundamental solution in an explicit analytical form 
by an inverse transformation of the frequency domain results, the convolution integral in the BIE can be numerically approximated by a new approach so-called "Operational Quadrature Methods" developed by Lubich [35, 36].

Finally, some numerical results are plotted that show the accuracy of the proposed solutions. The derived results are verified analytically by comparison with the previously introduced corresponding fundamental solutions in elastodynamic limiting case.

\section{Governing equations}

In order to have a fully coupled model of unsaturated soil, the effect of the suction change on the skeleton deformation and on the water and air permeabilities should be considered. On the other hand the influence of stress level and induced-strain on the degree of saturation and pore pressure dissipation must be taken into account as well. The mass conservation equations of water and air and the equilibrium equation of skeleton associated with water and air flow equations and constitutive relation form a complete set of field equations [14, 15, 23, 22].

\subsection{Basic concepts and kinematics}

An unsaturated porous medium is a medium composed of a deformable solid skeleton and a porous space filled by two fluids (water $(w)$ and air (a) ) (Fig.1).

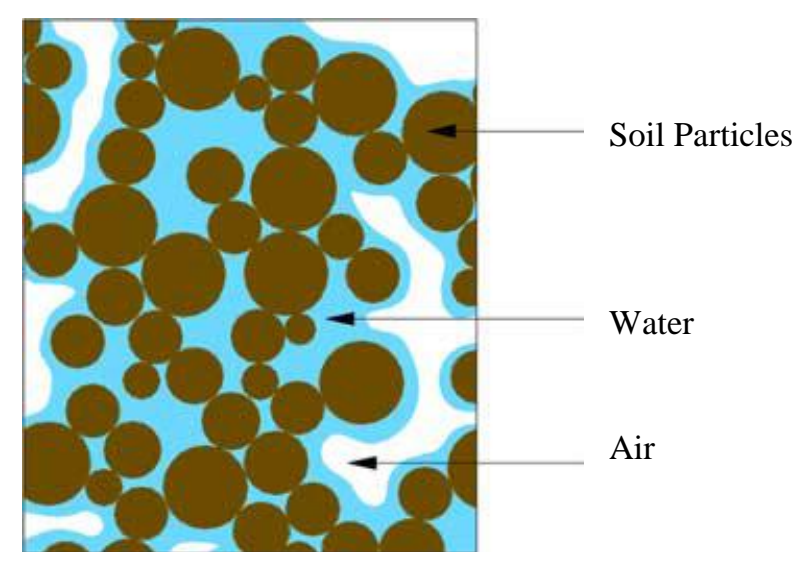

Figure 1. Unsaturated soil scheme 
The interconnected porous space is the space through which fluids mass exchanges occur. The remaining complementary space is the matrix. Hence, the matrix may be composed of both a solid part and a disconnected occluded space, whether saturated or not [10].

The displacement field is defined by the displacements of the solid skeleton $\mathbf{u}$ (or $u_{i}$ ) and the displacement of the fluids relative to the solid $\mathbf{w}^{\alpha}$ (or $w_{i}^{\alpha}$ ). The absolute displacement of the fluids $\mathbf{U}^{\alpha}$ (or $U_{i}^{\alpha}$ ) is defined in such a way that the volume of fluid $\alpha$ displaced through unit area normal to the $x_{i}$ direction is $n S_{\alpha} U_{i}^{\alpha}$ where $n$ is the porosity and $S_{\alpha}$ is the degree of saturation relative to fluid $\alpha$ and defined in a way that

$$
\begin{gathered}
S_{\alpha}=n_{\alpha} / n ; \quad \alpha=w, a \\
\sum_{\alpha} S_{\alpha}=1 \\
n=\sum_{\alpha} n S_{\alpha}
\end{gathered}
$$

\subsubsection{Skeleton Deformation}

The skeleton material point at time $t$, after deformation of the medium, is expressed by position vector $\mathbf{x}=\mathbf{x}(\mathbf{X}, t)$ in a Cartesian coordinate frame of orthonormal basis in which $\mathbf{X}$ is its initial position vector.

Deformation gradient of the solid skeleton $\mathbf{F}$ is defined by

$$
\mathbf{F}=\mathbf{I}+\nabla \mathbf{u}
$$

Where I is the second-order isotropic tensor with component $\delta_{i j}$, where $\delta_{i j}$ is the Kronecker delta. The symbol $\nabla(=\partial / \partial \mathbf{x})$ always stands for gradients with respect to $\mathbf{x}$ in this paper and $\mathbf{u}$ is the displacement vector of the skeleton whose initial and current positions are $\mathbf{X}$ and $\mathbf{x}$ $(\mathbf{u}=\mathbf{x}-\mathbf{X})$.

The Green-Lagrange strain tensor $\mathbf{E}$ which measures the deformation changes as a function of deformation gradient $\mathbf{F}$, in the limit of infinitesimal transformation reduces to the linearized strain tensor $\varepsilon$ : 


$$
\boldsymbol{\varepsilon}=\frac{1}{2}\left(\nabla \mathbf{u}+{ }^{t} \nabla \mathbf{u}\right)
$$

In infinitesimal transformation, the volume dilatation is written as:

$$
\operatorname{trace}(\boldsymbol{\varepsilon})=\varepsilon_{i i}=\nabla \cdot \mathbf{u}
$$

The observable volume dilatation of the skeleton is due to both the variation of the porous connected space and the volume dilatation of the matrix. The latter will be noted $\boldsymbol{\varepsilon}_{s}$, where the subscript $s$ stands for solid matrix. If $d \Omega^{s}$ and $d \Omega_{t}^{s}$ denote the volume occupied respectively, by the matrix, in the reference and current configuration, the volume dilatation of the matrix reads

$$
\varepsilon_{s}=\frac{d \Omega_{t}^{s}-d \Omega^{s}}{d \Omega^{s}}
$$

In this paper the assumption of incompressibility of the solid grain is considered. As a consequence $d \Omega_{t}^{s}=d \Omega^{s}$ and $\varepsilon_{s}=0$. Then, the volume dilatation of the skeleton is equal to the variation of the porous connected space $n$.

\subsubsection{Relative Flow Vector of a Fluid Volume}

In order to describe the material motion of an unsaturated porous medium, the movement of the $\alpha$ fluid particles relative to the initial configuration of the skeleton now needs to be specified. With that purpose in mind let $d \boldsymbol{\Gamma}$ be an infinitesimal skeleton material surface oriented by unit normal $\mathbf{n}(d \boldsymbol{\Gamma}=\mathbf{n} d \Gamma)$. At time $t$ and per unit of time, a $\alpha$ fluid volume written as

$$
q_{\alpha} d \Gamma=\dot{\mathbf{w}}^{\alpha} . \mathbf{n} d \Gamma ; \quad \alpha=w, a
$$

flows through this surface $d \boldsymbol{\Gamma}$ which is followed in the movement of the constituting skeleton particles. The term $q_{\alpha}$ represents the relative fluid volume flux per unit of surface area 
$\left[\mathrm{L}^{3} \cdot \mathrm{L}^{-2} \cdot \mathrm{T}^{-1}\right]$. Since $\mathbf{w}^{\alpha}\left(\right.$ or $\left.w_{i}^{\alpha}\right)$ is the displacement vector of the $\alpha$ fluid relative to the solid skeleton, the vector $\dot{\mathbf{w}}^{\alpha}(\mathbf{x}, t)$ is the Eulerian relative flow vector of fluid volume (with respect to the skeleton) or Darcy flow velocity for $\alpha$ fluid. Next, let $\mathbf{v}^{\alpha, s}(\mathbf{x}, t)$ be the $\alpha$ fluid particle velocity relative to the skeleton particle. From a kinematical point of view, the velocity $\mathbf{v}^{\alpha, s}(\mathbf{x}, t)$ can be written as

$$
\mathbf{v}^{\alpha, s}=\mathbf{v}^{\alpha}-\mathbf{v}^{s} ; \quad \alpha=w, a
$$

Where $\mathbf{v}^{\alpha}(\mathbf{x}, t)$ is the Eulerian absolute fluid velocity and it is equal to $\dot{\mathbf{U}}^{\alpha}$.

Here, the relative velocity $\mathbf{v}^{\alpha, s}(\mathbf{x}, t)$ can be defined from the relative flow vector of fluid volume $\dot{\mathbf{w}}^{\alpha}$ through the relation

$$
\dot{\mathbf{w}}^{\alpha}=n_{\alpha} \mathbf{v}^{\alpha, s}=n S_{\alpha} \mathbf{v}^{\alpha, s}=n S_{\alpha}\left(\dot{\mathbf{U}}^{\alpha}-\dot{\mathbf{u}}\right)
$$

\subsection{Mass Conservation Equation:}

\subsubsection{Mass Conservation of Solid Skeleton}

The mass of the solid skeleton in a representative elementary volume can be written as

$$
M_{s}=\int_{\Omega_{t}}(1-n) \rho_{s} d \Omega_{t}
$$

Where $(1-n) \rho_{s}$ represents the volume density of solid skeleton mass in the current configuration;

The mass conservation requires that 


$$
\begin{aligned}
\frac{\mathrm{d} M_{s}}{\mathrm{~d} t} & =\frac{\mathrm{d}}{\mathrm{d} t} \int_{\Omega_{t}}(1-n) \rho_{s} d \Omega_{t} \\
& =\int_{\Omega_{t}}\left(\frac{\partial(1-n) \rho_{s}}{\partial t}+\operatorname{div}\left[(1-n) \rho_{s} \otimes \mathbf{v}^{\mathrm{s}}\right]\right) d \Omega_{t}=0
\end{aligned}
$$

Where $\operatorname{div}\left(g \otimes \mathbf{v}^{\mathbf{s}}\right)=g \cdot \operatorname{div} \mathbf{v}^{\mathrm{s}}+\operatorname{grad} g \cdot \mathbf{v}^{\mathbf{s}} ;$

Then we have

$$
(1-n) \frac{\mathrm{d} \rho_{s}}{\mathrm{~d} t}-\rho_{s} \frac{\mathrm{d} n}{\mathrm{~d} t}+(1-n) \rho_{s} \operatorname{div} \mathbf{v}^{\mathrm{s}}=0
$$

By the hypotheses of the incompressibility of the soil grains $\left(\mathrm{d} \rho_{s} / \mathrm{d} t\right)$, we obtain

$$
\frac{\mathrm{d} n}{\mathrm{~d} t}=(1-n) \operatorname{div} \mathbf{v}^{\mathrm{s}}=(1-n) \dot{u}_{i, i}
$$

This equation states that the deformation of the solid skeleton consists in the rearrangement of the grains only.

\subsubsection{Mass Conservation of Water}

The mass of the water in a representative elementary volume can be written as

$$
M_{w}=\int_{\Omega_{t}} n_{w} \rho_{w} d \Omega_{t}=\int_{\Omega_{t}} n S_{w} \rho_{w} d \Omega_{t}
$$

Where $n_{w} \rho_{w}$ represents the volume density of water mass in the current configuration;

The mass conservation requires that

$$
\begin{aligned}
\frac{\mathrm{d}^{\mathrm{w}} M_{w}}{\mathrm{~d} t} & =\frac{\mathrm{d}^{\mathrm{w}}}{\mathrm{d} t} \int_{\Omega_{t}} n S_{w} \rho_{w} d \Omega_{t} \\
& =\int_{\Omega_{t}}\left(\frac{\partial n S_{w} \rho_{w}}{\partial t}+\operatorname{div}\left[n S_{w} \rho_{w} \otimes \mathbf{v}^{\mathrm{w}}\right]\right) d \Omega_{t}=0
\end{aligned}
$$


By introducing equations (7) and (8) into (14):

$$
\frac{\mathrm{d}^{\mathrm{w}} M_{w}}{\mathrm{~d} t}=\int_{\Omega_{t}}\left(\frac{\partial n S_{w} \rho_{w}}{\partial t}+\operatorname{div}\left[n S_{w} \rho_{w} \otimes \mathbf{v}^{\mathrm{s}}+\rho_{w} \otimes \dot{\mathbf{w}}^{w}\right]\right) d \Omega_{t}=0
$$

Since equation (15) must hold for any volume $\Omega_{t}$, the integrant must be zero, giving the water fluid mass local balance equation commonly called the water fluid continuity equation. By supposing a homogenous medium that satisfies $\operatorname{grad}\left(\rho_{w}\right)=0$ it reads

$$
n S_{w} \frac{\mathrm{d} \rho_{w}}{\mathrm{~d} t}+n \rho_{w} \frac{\mathrm{d} S_{w}}{\mathrm{~d} t}+S_{w} \rho_{w} \frac{\mathrm{d} n}{\mathrm{~d} t}+n S_{w} \rho_{w} \dot{u}_{i, i}^{s}+\rho_{w} \dot{w}_{i, i}^{w}=0
$$

By the state equation of variation of porosity, equation (12), we obtain

$$
\frac{n S_{w}}{\rho_{w}} \frac{\mathrm{d} \rho_{w}}{\mathrm{~d} t}+n \frac{\mathrm{d} S_{w}}{\mathrm{~d} t}+S_{w} \dot{u}_{i, i}+\dot{w}_{i, i}^{w}=0
$$

The first part in the left hand side of the equation (17) can be written as

$$
\frac{1}{\rho_{w}} \frac{\mathrm{d} \rho_{w}}{\mathrm{~d} t}=\frac{1}{\rho_{w}} \frac{\mathrm{d} \rho_{w}}{\mathrm{~d} p_{w}} \frac{\mathrm{d} p_{w}}{\mathrm{~d} t}=C_{w} \frac{\mathrm{d} p_{w}}{\mathrm{~d} t}
$$

Where $C_{w}=\frac{\left(\mathrm{d} \rho_{w} / \rho_{w}\right)}{\mathrm{d} p_{w}}$ is the compressibility of water.

Then,

$$
C_{w} n S_{w} \frac{\mathrm{d} p_{w}}{\mathrm{~d} t}+n \frac{\mathrm{d} S_{w}}{\mathrm{~d} t}+S_{w} \dot{u}_{i, i}+\dot{w}_{i, i}^{w}=0
$$

As mentioned later $\mathrm{d} S_{w} / \mathrm{d} t$ in equation (19) can be written as: 


$$
\frac{\mathrm{d} S_{w}}{\mathrm{~d} t}=g_{1} \frac{\mathrm{d}\left(p_{a}-p_{w}\right)}{\mathrm{d} t}
$$

Where $g_{1}=\frac{\mathrm{d} S_{w}}{\mathrm{~d}\left(p_{a}-p_{w}\right)}$

Then final mass equation takes this form:

$$
\dot{w}_{i, i}^{w}=-S_{w} \dot{\varepsilon}_{i i}+C_{w w} \dot{p}_{w}+C_{w a} \dot{p}_{a}
$$

Where $C_{w w}=\left(n g_{1}-C_{w} n S_{w}\right) ; \quad C_{w a}=C_{a w}=-n g_{1}$

\subsubsection{Mass Conservation of air}

With the same approach presented for the water mass conservation, the mass conservation equation of the air in a representative elementary volume can be written as

$$
C_{a} n S_{a} \frac{\mathrm{d} p_{a}}{\mathrm{~d} t}+n \frac{\mathrm{d} S_{a}}{\mathrm{~d} t}+S_{a} \dot{u}_{i, i}+\dot{w}_{i, i}^{a}=0
$$

Where $C_{a}=\frac{\left(\mathrm{d} \rho_{a} / \rho_{a}\right)}{\mathrm{d} p_{a}}$;

By using equation (1b), $S_{w}+S_{a}=1$, it is obtained

$$
\frac{\mathrm{d} S_{a}}{\mathrm{~d} t}=-\frac{\mathrm{d} S_{w}}{\mathrm{~d} t}=-g_{1} \frac{\mathrm{d}\left(p_{a}-p_{w}\right)}{\mathrm{d} t}
$$

Then the final air mass equation can be written as

$$
\dot{w}_{i, i}^{a}=-S_{a} \dot{\varepsilon}_{i i}+C_{w a} \dot{p}_{w}+C_{a a} \dot{p}_{a}
$$

Where $C_{a a}=\left(n g_{1}-C_{a} n S_{a}\right) ; \quad C_{w a}=C_{a w}=-n g_{1}$ 


\subsection{Equilibrium Equation:}

The total equation of motion for a unit element of porous medium can be written as

$$
\nabla \cdot(\boldsymbol{\sigma})+\rho \mathbf{g}=\rho \frac{\mathrm{d} \mathbf{v}^{\mathbf{s}}}{\mathrm{d} t}+\rho_{w} n S_{w}\left(\frac{\mathrm{d} \mathbf{v}^{\mathrm{w}, \mathbf{s}}}{\mathrm{d} t}+\operatorname{grad}\left(\mathbf{v}^{\mathbf{w}}\right) \cdot \mathbf{v}^{\mathbf{w}, \mathbf{s}}\right)+\rho_{a} n S_{a}\left(\frac{\mathrm{d} \mathbf{v}^{\mathbf{a}, \mathbf{s}}}{\mathrm{d} t}+\operatorname{grad}\left(\mathbf{v}^{\mathbf{a}}\right) \cdot \mathbf{v}^{\mathrm{a}, \mathbf{s}}\right)
$$

The convective acceleration terms in the above equations are neglected because of numerical difficulties, but relative acceleration terms are retained [44]. If the relative acceleration terms of the fluids ( $\ddot{\mathbf{w}}^{\mathbf{w}}$ and $\ddot{\mathbf{w}}^{\mathbf{a}}$ ) are omitted, we have simply

$$
\nabla \cdot(\boldsymbol{\sigma})+\rho \mathbf{g}=\rho \ddot{\mathbf{u}}
$$

The equilibrium equation for the skeleton can be written in indicial notation as follows:

$$
\left(\sigma_{i j}-\delta_{i j} p_{a}\right)_{, j}+p_{a, i}+f_{i}=\rho \ddot{u}_{i}
$$

\subsection{Flow Equation for the Water:}

Based on generalized Darcy's law for describing the balance of the forces acting on the liquid phase of the representative elementary volume, namely the gradient of suction, the inertia and the resistant force of water due to its viscosity, the water velocity in the unsaturated soil takes the following form:

$$
n_{w} \mathbf{v}^{\mathbf{w}, \mathbf{s}}=k_{w}\left(-\nabla p_{w}+\rho_{w} \mathbf{g}-\rho_{w}\left[\frac{\mathrm{d} \mathbf{v}^{\mathbf{s}}}{\mathrm{d} t}+\frac{\mathrm{d} \mathbf{v}^{\mathbf{w}, \mathbf{s}}}{\mathrm{d} t}+\mathbf{v}^{\mathbf{w}, \mathbf{s}} \nabla \mathbf{v}^{\mathbf{w}}\right]\right)
$$


In which $k_{w}=a_{w} 10^{e \alpha_{w}}\left(\left(S_{w}-S_{w u}\right) /\left(1-S_{w u}\right)\right)^{d_{w}}$ denotes the water permeability in an unsaturated soil (where $e$ is the void ratio, $a_{w}, \alpha_{w}, d_{w}$ and $S_{w u}$ are constants depending on the soil studied).

By neglecting the convective acceleration terms in the above equations, it is obtained

$$
\dot{\mathbf{w}}^{\mathbf{w}}=k_{w}\left(-\nabla p_{w}+\rho_{w} \mathbf{g}-\rho_{w} \ddot{\mathbf{u}}-\frac{\rho_{w}}{n_{w}} \ddot{\mathbf{w}}^{\mathbf{w}}\right)
$$

By omitting the relative acceleration terms of the water it is obtained:

$$
-p_{w, i}=\rho_{w} \ddot{\mathbf{u}}+\frac{\dot{\mathbf{w}}^{\mathbf{w}}}{k_{w}}-\rho_{w} \mathbf{g}
$$

\subsection{Flow Equation for the Air:}

With the same approach presented for the water based on generalized Darcy's law, the air velocity in the unsaturated soil takes the following form:

$$
-p_{a, i}=\rho_{a} \ddot{\mathbf{u}}+\frac{\dot{\mathbf{w}}^{\mathbf{a}}}{k_{a}}-\rho_{a} \mathbf{g}
$$

In which $k_{a}=\left(c_{a} \gamma_{a} / \mu_{a}\right)\left(e\left(1-S_{w}\right)\right)^{d_{a}}$ is the air permeability (where $\mu_{a}$ is the air viscosity, $e$ is the void ratio, $c_{a}$ and $d_{a}$ are constants depending on the soil studied).

\subsection{Constitutive Law for the Solid Skeleton:}

Fully coupled formulation of unsaturated soil can be obtained by considering two constitutive laws in order to model the effects of suction on deformation and saturation. The first one is the stress-suction-strain relation which considers the effect of suction on strain. The second 
one is the stress-suction-saturation which describes the evolution of saturation under the effects stress level and variation of suction [15].

The first constitutive law of the skeleton (stress-suction-strain relation) can therefore be defined incrementally in terms of total net stress changes as:

$$
d\left(\sigma_{i j}-\delta_{i j} p_{a}\right)=D_{i j k l}\left(d \varepsilon_{k l}^{\sigma}\right)
$$

In the above, $d\left(\sigma_{i j}-\delta_{i j} p_{a}\right)$ is the total net stress increment, $D_{i j k l}$ is the stress-strain relationship tensor that is a function of constitutive model of soils. $d \varepsilon_{k l}^{\sigma}$ is the strain increment due to total net stress changes in unsaturated soil. It can be supposed that:

$$
\mathrm{d} \boldsymbol{\varepsilon}^{\sigma}=\mathrm{d} \boldsymbol{\varepsilon}-\mathrm{d} \boldsymbol{\varepsilon}^{\text {suc }}
$$

Where $\mathrm{d} \boldsymbol{\varepsilon}^{\text {suc }}$ and $\mathrm{d} \boldsymbol{\varepsilon}$ are volumetric strain increment due to suction changes and total strain increment, respectively.

By introducing equation (33) into (32), it is obtained:

$$
d\left(\sigma_{i j}-\delta_{i j} p_{a}\right)=D_{i j k l}\left(d \varepsilon_{k l}-d \varepsilon_{k l}^{s u c}\right)
$$

In this equation $d \varepsilon_{k l}^{s u c}$ can be written as follows:

$$
d\left(p_{a}-p_{w}\right) \delta_{k l}=D_{k l}^{s u c} d \varepsilon_{k l}^{s u c} \Rightarrow d \varepsilon_{k l}^{s u c}=\left(D_{k l}^{s u c}\right)^{-1} d\left(p_{a}-p_{w}\right) \delta_{k l}
$$

And also by substituting equation (35) into equation (34), it is obtained:

$$
d\left(\sigma_{i j}-\delta_{i j} p_{a}\right)=D_{i j k l} \mathrm{~d} \varepsilon_{k l}-F_{i j}^{s} d\left(p_{a}-p_{w}\right)
$$

Where $\underline{F}^{s}=\underline{D} \cdot \underline{D}^{\text {suc-1 }}$.

Therefore, by introducing the elasticity matrix $\mathbf{D}$ into equation (36), the constitutive law is written as: 


$$
d\left(\sigma_{i j}-\delta_{i j} p_{a}\right)=\left(\lambda \delta_{i j} d \varepsilon_{k k}+2 \mu d \varepsilon_{i j}\right)-F_{i j}^{s} d\left(p_{a}-p_{w}\right)
$$

By integrating this equation, (37) can be written as

$$
\left(\sigma_{i j}-\delta_{i j} p_{a}\right)=\left(\lambda \delta_{i j} \varepsilon_{k k}+2 \mu \varepsilon_{i j}\right)-F_{i j}^{s}\left(p_{a}-p_{w}\right)
$$

\subsubsection{State surface of void ratio}

As shown before in equation (36) the suction modulus matrix $\mathbf{F}^{s}$ is

$$
F^{s}=\underline{\underline{D}} \cdot \underline{D}^{\text {suc-1 }}
$$

Where $\underline{D}^{\text {suc }}$ is a vector obtained from the state surface of void ratio $(e)$ which is a function of the independent variables

$$
\begin{gathered}
e=\mathrm{f}\left(\left(\sigma-p_{a}\right),\left(p_{a}-p_{w}\right)\right) \\
d \varepsilon^{s u c}=D^{s u c-1} d\left(p_{a}-p_{w}\right)
\end{gathered}
$$

Where

$$
D^{s u c-1}=\frac{1}{1+e} \frac{\partial e}{\partial\left(p_{a}-p_{w}\right)}
$$

The elasticity matrix $(\underline{\underline{D}})$ can be presented by using the bulk modulus and the tangent modulus

$$
\underline{\underline{D}}=\underline{\underline{D}}\left(K_{0}, E_{t}\right)=\underline{\underline{D}}\left(\sigma-p_{a}, p_{a}-p_{w}\right)
$$

Where $E_{t}$ is tangent elastic modulus which can be evaluated as 


$$
E_{t}=E_{l}+E_{s}
$$

Where $E_{l}$ is the elastic modulus in absence of suction and

$$
E_{s}=m_{s}\left(p_{a}-p_{w}\right)
$$

$m_{s}$ being a constant, $E_{s}$ represents the effect of suction on the elastic modulus.

$K_{0}$ is the bulk modulus of an open system and evaluated from the surface state of void ratio (e)

$$
\begin{gathered}
\mathrm{d} \varepsilon_{v}=\frac{1}{1+e} \frac{\partial e}{\partial\left(\sigma-p_{a}\right)} \mathrm{d}\left(\sigma-p_{a}\right)=K_{0}^{-1} \mathrm{~d}\left(\sigma-p_{a}\right) \\
K_{0}=(1+e)\left(\frac{\partial e}{\partial\left(\sigma-p_{a}\right)}\right)^{-1}
\end{gathered}
$$

In order to assure the compatibility with linear law, the volumetric variation module must be defined so that the volume change properties of unsaturated soil under increasing monotonic variation of degree of saturation should be satisfied:

$$
K_{0}=\frac{K_{0}^{L}}{a-b S / \sigma_{e}}
$$

Where $K_{0}^{L}$ is the bulk modulus in absence of suction and $a, b$ are constants, $\sigma_{e}$ is the swelling pressure and $S$ stands for suction $\left(p_{a}-p_{w}\right)$. For a suction equal to zero and setting $a=1$, one obtains the bulk modulus in the linear elastic model

$$
K_{0}=K_{0}^{L}
$$

By using this relation, and integrating in relation to the stress and considering the influence of the suction, the final form of state surface for void ratio [14] is simplified to: 


$$
e=\frac{\left(1+e_{0}\right)}{\exp \left[\frac{a\left(\sigma-p_{a}\right)+b\left(p_{a}-p_{w}\right)\left(1-\left(\sigma-p_{a}\right) / \sigma_{e}\right)}{K_{0}^{L}}\right]}-1
$$

It can be observed that with a suction and stress equal zero, $e=e_{0}$. Now, by using the new formulation of the state surface, $\underline{D}^{\text {suc-1 }}$ vector can be obtained as

$$
D^{s u c-1}=\frac{1}{1+e} \frac{\partial e}{\partial\left(p_{a}-p_{w}\right)}=\frac{b}{K_{0}^{L}}\left(1-\frac{\left(\sigma-p_{a}\right)}{\sigma_{e}}\right)
$$

\subsubsection{State surface of degree of saturation}

The second constitutive law (stress-suction-saturation relation) which describes the evolution of water saturation under the effects of stress level and variation of suction is written as follows

$$
S_{w}=f\left(\left(\sigma-p_{a}\right),\left(p_{a}-p_{w}\right)\right)
$$

Numerous relations have been introduced to define the degree of saturation of unsaturated soils, but the logarithmic form based on suction variations is one of the most common and reliable ones. The exponential form of the degree of saturation is found here by omitting the dependency to the net stress in the original equation [14]:

$$
S_{w}=1-\left\{1-\exp \left(\beta_{w}\left(p_{a}-p_{w}\right)\right)\right\}
$$

In which $\beta_{w}$ is constant. By assuming a negative $\beta_{w}$, one can see that any increase in suction results a decrease in $S_{w}$ and any decrease in suction results the approach of $S_{w}$ to one (saturated).

As shown in Figure 2, this relation has good agreement with the expression of the state surface of degree of saturation proposed by Gens et al. (1997) [27]. This expression is based 
on the experimental studies carried out by Villar and Martin (1993) [50] and is obtained by adopting Van Genutchen model.

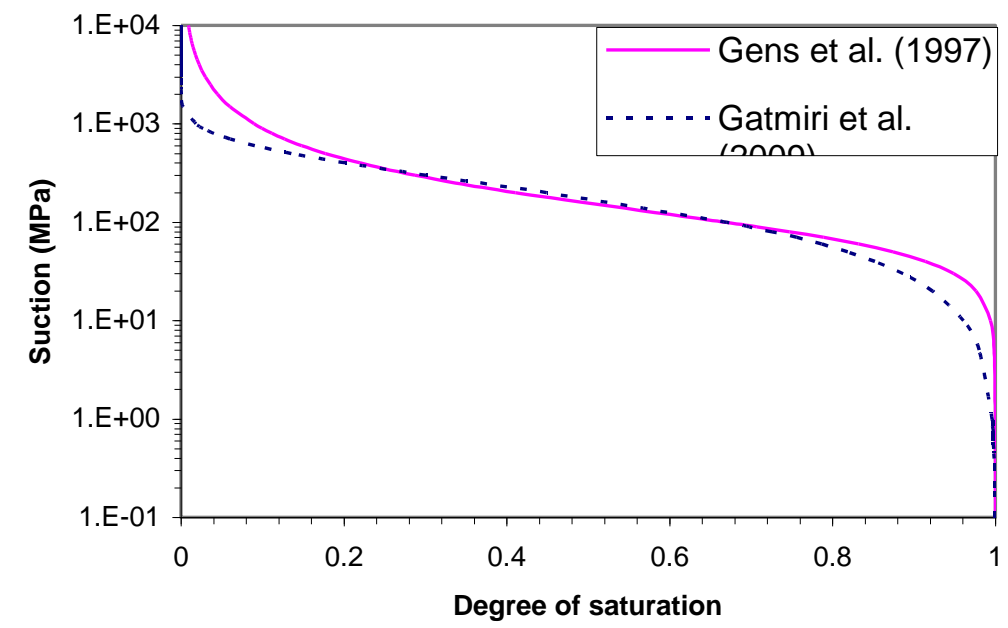

Figure 2. Comparison between the state surface of degree of saturation proposed by Gatmiri (1997) and Gens et al. (1997)

\subsection{Summery of the Field Equations:}

Then, briefly by neglecting the body forces of fluids, the governing equations are written as below for the unknowns solid displacement, water pore pressure and air pore pressure:

\section{Equilibrium equation:}

$$
\left(\sigma_{i j}-\delta_{i j} p_{a}\right)_{, j}+p_{a, i}+f_{i}=\rho \ddot{u}_{i}
$$

Constitutive law for the solid skeleton:

$$
\left(\sigma_{i j}-\delta_{i j} p_{a}\right)=\left(\lambda \delta_{i j} \varepsilon_{k k}+2 \mu \varepsilon_{i j}\right)-F_{i j}^{s}\left(p_{a}-p_{w}\right)
$$

Mass conservation equation for water:

$$
\dot{w}_{i, i}^{w}=-S_{w} \dot{\varepsilon}_{i i}+C_{w w} \dot{p}_{w}+C_{w a} \dot{p}_{a}
$$


Flow equation for water:

$$
\dot{w}_{i}^{w}=k_{w}\left(-p_{w, i}-\rho_{w} \ddot{u}_{i}\right)
$$

Mass conservation equation for air:

$$
\dot{w}_{i, i}^{a}=-S_{a} \dot{\varepsilon}_{i i}+C_{w a} \dot{p}_{w}+C_{a a} \dot{p}_{a}
$$

Flow Equation for air:

$$
\dot{w}_{i}^{a}=k_{a}\left(-p_{a, i}-\rho_{a} \ddot{u}_{i}\right)
$$

By introducing (38.rep) into (27.rep), (30.rep) into (21.rep) and (31.rep) into (24.rep), we have

$$
\begin{gathered}
(\lambda+\mu) u_{\beta, \alpha \beta}+\mu u_{\alpha, \beta \beta}+F^{s} p_{w, \alpha}+\left(1-F^{s}\right) p_{a, \alpha}-\rho \ddot{u}_{\alpha}+f_{\alpha}=0 \\
-S_{w} \dot{u}_{\alpha, \alpha}+\rho_{w} k_{w} \ddot{u}_{\alpha, \alpha}+k_{w} p_{w, \alpha \alpha}+C_{w w} \dot{p}_{w}+C_{w a} \dot{p}_{a}=0 \\
-S_{a} \dot{u}_{\alpha, \alpha}+\rho_{a} k_{a} \ddot{u}_{\alpha, \alpha}+k_{a} p_{a, \alpha \alpha}+C_{w a} \dot{p}_{w}+C_{a a} \dot{p}_{a}=0
\end{gathered}
$$

\subsection{Governing Equations in Laplace Transformed Domain}

One of the most common and straightforward methods for eliminating the time variable of a partial differential equation is to apply the Laplace transform. In this manner, after solving the differential equation in Laplace transform domain, one can obtain the time domain solution by applying an inverse Laplace transform on the Laplace transform domain solution [19]. We remember that [1]. 


$$
\begin{gathered}
L\{f(x, t)\}=\tilde{f}(x, s)=\int_{0}^{\infty} e^{-s t} f(x, t) d t \\
L\left\{f^{(n)}(x, t)\right\}=s^{n} \tilde{f}(x, s)-s^{n-1} f(x, 0)-s^{n-2} f^{\prime}(x, 0)-\ldots-f^{(n-1)}(x, 0)
\end{gathered}
$$

And assuming

$$
\begin{gathered}
u_{i(t=0)}=w_{i(t=0)}^{w}=w_{i(t=0)}^{a}=0 \\
p_{w(t=0)}=p_{a(t=0)}=0
\end{gathered}
$$

Eqs (54), (55) and (56) will be reduced to

$$
\begin{gathered}
(\lambda+\mu) \tilde{u}_{\beta, \alpha \beta}+\tilde{\mu} u_{\alpha, \beta \beta}+F^{s} \tilde{p}_{w, \alpha}+\left(1-F^{s}\right) \tilde{p}_{a, \alpha}-\rho s^{2} \tilde{u}_{\alpha}+\tilde{f}_{\alpha}=0 \\
-s \theta_{1} \tilde{u}_{\alpha, \alpha}+k_{w} \tilde{p}_{w, \alpha \alpha}+C_{w w} s \tilde{p}_{w}+C_{w a} s \tilde{p}_{a}=0 \\
-s \theta_{2} \tilde{u}_{\alpha, \alpha}+C_{w a} s \tilde{p}_{w}+k_{a} \tilde{p}_{a, \alpha \alpha}+C_{a a} s \tilde{p}_{a}=0
\end{gathered}
$$

Where $\theta_{1}=\left(S_{w}-\rho_{w} \cdot k_{w} \cdot s\right)$ and $\theta_{2}=\left(S_{a}-\rho_{a} \cdot k_{a} \cdot s\right)$.

We rewrite compactly the transformed coupled differential equation system Eqs (59), (60) and (61) into the following matrix form:

$$
\tilde{\mathbf{B}}\left[\begin{array}{c}
\tilde{u}_{i} \\
\tilde{p}_{w} \\
\tilde{p}_{a}
\end{array}\right]+\left[\begin{array}{c}
\tilde{f}_{i} \\
0 \\
0
\end{array}\right]=\mathbf{0}
$$

With the not self-adjoint operator $\tilde{\mathbf{B}}$ :

$$
\tilde{\mathbf{B}}=\left[\begin{array}{ccc}
\left(\mu \Delta-\rho s^{2}\right) \delta_{i j}+(\lambda+\mu) \partial_{i} \partial_{j} & F^{s} \partial_{i} & \left(1-F^{s}\right) \partial_{i} \\
-s \theta_{1} \partial_{j} & k_{w} \Delta+C_{w w} s & C_{w a} s \\
-s \theta_{2} \partial_{j} & C_{w a} s & k_{a} \Delta+C_{a a} s
\end{array}\right]
$$


With $i, j$ from one to four in two dimensional problems. In equation (63), the partial derivative ()$_{i}$ is denoted by $\partial_{i}$ and $\Delta=\partial_{i i}$ is the Laplacian operator.

Based on this equation in the next section boundary integral equation and fundamental solutions are derived.

\section{Boundary integral equation}

To the authors' knowledge, the boundary integral equations for dynamic unsaturated poroelasticity have not yet been obtained. It is aimed to achieve to these integral equations at such a level that it allows application to physical meaningful problem. The corresponding fundamental solutions will be derived in section 4. Along with Boundary Element Method permits an easy discretization and implementation in a numerical code. To that end the present section is dedicated to the derivation of a set of the boundary integral equations for dynamic multiphase poroelasticity using the weighted residuals method. In this method, the poroelastodynamic integral equation is derived directly by equating the inner product of Eqs. (59), (60) and (61), written in matrix form with operator matrix $\tilde{\mathbf{B}}$ defined in equation (63), and the matrix of the adjoint fundamental solutions $\tilde{\mathbf{G}}^{*}$ implying that

$$
\tilde{\mathbf{B}}^{*} \tilde{\mathbf{G}}^{*}+\mathbf{I} \delta(x-\xi)=0
$$

to a null vector, i.e.

$$
\int_{\Omega} \tilde{\mathbf{B}}\left[\begin{array}{c}
\tilde{u}_{\alpha} \\
\tilde{p}_{w} \\
\tilde{p}_{a}
\end{array}\right] \tilde{\mathbf{G}}^{*} \mathrm{~d} \Omega=0 \quad \text { with } \quad \tilde{\mathbf{G}}^{*}=\left[\begin{array}{lll}
\tilde{G}_{\alpha \beta}^{*} & \tilde{G}_{\alpha 3}^{*} & \tilde{G}_{\alpha 4}^{*} \\
\tilde{G}_{3 \beta}^{*} & \tilde{G}_{33}^{*} & \tilde{G}_{34}^{*} \\
\tilde{G}_{4 \beta}^{*} & \tilde{G}_{43}^{*} & \tilde{G}_{44}^{*}
\end{array}\right]=\left[\begin{array}{ccc}
\tilde{U}_{\alpha \beta}^{s *} & \tilde{U}_{\alpha}^{w *} & \tilde{U}_{\alpha}^{a *} \\
\tilde{P}_{w \beta}^{s *} & \tilde{P}_{w *}^{w^{*}} & \tilde{P}_{w}^{a^{*}} \\
\tilde{P}_{a \beta}^{s *} & \tilde{P}_{a}^{w *} & \tilde{P}_{a}^{a *}
\end{array}\right]
$$

where the integration is performed over a domain $\Omega$ with boundary $\Gamma$ and vanishing body forces and sources are assumed. By this inner product, essentially, the error in satisfying the governing differential equations (59), (60) and (61), is forced to be orthogonal to $\tilde{\mathbf{G}}^{*}$ [47]. The equation (65) written in index notation becomes 


$$
\begin{aligned}
\int_{\Omega} & {\left[(\lambda+\mu) \tilde{G}_{\alpha j}^{*} \tilde{u}_{\beta, \beta \alpha}+\mu \tilde{G}_{\alpha j}^{*} \Delta \tilde{u}_{\alpha}-\rho s^{2} \tilde{u}_{\alpha} \tilde{G}_{\alpha j}^{*}+F_{s} \tilde{G}_{\alpha j}^{*} \tilde{p}_{w, \alpha}+\left(1-F_{s}\right) \tilde{G}_{\alpha j}^{*} \tilde{p}_{a, \alpha}\right.} \\
& +k_{w} \tilde{G}_{3 j}^{*} \Delta \tilde{p}_{w}-s \theta_{1} \tilde{G}_{3 j}^{*} \tilde{u}_{\alpha, \alpha}+C_{w w} s \tilde{G}_{3 j}^{*} \tilde{p}_{w}+C_{w a} s \tilde{G}_{3 j}^{*} \tilde{p}_{a} \\
& \left.+k_{a} \tilde{G}_{4 j}^{*} \Delta \tilde{p}_{a}-s \theta_{2} \tilde{G}_{4 j}^{*} \tilde{u}_{\alpha, \alpha}+C_{w a} s \tilde{G}_{4 j}^{*} \tilde{p}_{w}+C_{a a} s \tilde{G}_{4 j}^{*} \tilde{p}_{a}\right] \mathrm{d} \Omega=0
\end{aligned}
$$

where $\alpha, \beta=1,2$ and $i, j=1,4$. For each term in Eq (66), integrating by parts twice over the domain using the theory of Green's formula and using partial integration, the operator $\tilde{\mathbf{B}}$ is transformed from acting on the vector of unknowns $\left[\begin{array}{lll}\tilde{u}_{\alpha} & \tilde{p}_{w} & \tilde{p}_{a}\end{array}\right]^{T}$ to the matrix of fundamental solutions $\mathbf{G}^{* *}$.

To show the principal procedure, five exemplary parts of integral equation (66) are presented in detail. The remaining partial integrations for the other parts in integral equation (66) can be performed analogously.

$$
\begin{aligned}
& \lambda \int_{\Omega} \tilde{G}_{\alpha j}^{*} \tilde{u}_{\beta, \beta \alpha} \mathrm{d} \Omega=\lambda \int_{\Gamma} \tilde{G}_{\alpha j}^{*} \tilde{u}_{k, k} n_{\beta} \delta_{\alpha \beta} \mathrm{d} \Gamma-\lambda \int_{\Gamma} \tilde{u}_{\alpha} \tilde{G}_{k j, k}^{*} n_{\beta} \delta_{\alpha \beta} \mathrm{d} \Gamma+\lambda \int_{\Omega} \tilde{u}_{\alpha} \tilde{G}_{\beta j, \alpha \beta}^{*} \mathrm{~d} \Omega \\
& \mu \int_{\Omega} \tilde{G}_{\alpha j}^{*} \Delta \tilde{u}_{\alpha} \mathrm{d} \Omega=\mu \int_{\Gamma}\left(\tilde{G}_{\alpha j}^{*} \tilde{u}_{\alpha, \beta}-\tilde{u}_{\alpha} \tilde{G}_{\alpha j, \beta}^{*}\right) n_{\beta} \mathrm{d} \Gamma+\mu \int_{\Omega} \Delta \tilde{G}_{\beta j}^{*} \tilde{u}_{\alpha} \mathrm{d} \Omega \\
& F_{s} \int_{\Omega} \tilde{G}_{\alpha j}^{*} \tilde{p}_{w, \alpha} \mathrm{d} \Omega=F_{s} \int_{\Gamma} \tilde{G}_{\alpha j}^{*} \tilde{p}_{w} n_{\beta} \delta_{\alpha \beta} \mathrm{d} \Gamma-F_{s} \int_{\Omega} \tilde{G}_{\alpha j, \alpha}^{*} \tilde{p}_{w} \mathrm{~d} \Omega \\
& k_{w} \int_{\Omega} \tilde{G}_{3 j}^{*} \Delta \tilde{p}_{w} \mathrm{~d} \Omega=k_{w} \int_{\Gamma} \tilde{G}_{3 j}^{*} \tilde{p}_{w, n} \mathrm{~d} \Gamma-k_{w} \int_{\Gamma} \tilde{G}_{3 j, n}^{*} \tilde{p}_{w} \mathrm{~d} \Gamma+k_{w} \int_{\Omega} \Delta \tilde{G}_{3 j}^{*} \tilde{p}_{w} \mathrm{~d} \Omega \\
& -s \theta_{1} \int_{\Omega} \tilde{G}_{3 j}^{*} \tilde{u}_{\alpha, \alpha} \mathrm{d} \Omega=-s \theta_{1} \int_{\Gamma} \tilde{G}_{3 j}^{*} \tilde{u}_{\alpha} n_{\beta} \delta_{\alpha \beta} \mathrm{d} \Gamma+s \theta_{1} \int_{\Omega} \tilde{u}_{\alpha} \tilde{G}_{3 j, \alpha}^{*} \mathrm{~d} \Omega
\end{aligned}
$$

As shown in Eqs. (67a) to (67e), the integrals with one differentiation lead to the changes in the sign of the resulting domain integral while it remains unchanged in the case of two integration by parts [46]. This leads the transformation of the operator $\tilde{\mathbf{B}}$ into its adjoint operator $\tilde{\mathbf{B}}^{*}$.

This yields the following system of integral equations in index notation as

$$
\begin{aligned}
& \int_{\Gamma}\left[\left(\lambda \tilde{u}_{k, k}-F_{s}\left(\tilde{p}_{a}-\tilde{p}_{w}\right)+\tilde{p}_{a}\right) n_{\beta} \delta_{\alpha \beta}+\mu\left(\tilde{u}_{\beta, \alpha}+\tilde{u}_{\alpha, \beta}\right) n_{\beta}\right] \tilde{G}_{\alpha j}^{*} \mathrm{~d} \Gamma \\
& -\int_{\Gamma} \tilde{u}_{\alpha}\left[\left(\lambda \tilde{G}_{k j, k}^{*}+s \theta_{1} \tilde{G}_{3 j}^{*}+s \theta_{2} \tilde{G}_{4 j}^{*}\right) n_{\beta} \delta_{\alpha \beta}+\mu\left(\tilde{G}_{\alpha j, \beta}^{*}+\tilde{G}_{\beta j, \alpha}^{*}\right)\right] \mathrm{d} \Gamma \\
& +k_{w} \int_{\Gamma}\left(\tilde{p}_{w, n} \tilde{G}_{3 j}^{*}-\tilde{p}_{w} \tilde{G}_{3 j, n}^{*}\right) \mathrm{d} \Gamma+k_{a} \int_{\Gamma}\left(\tilde{p}_{a, n} \tilde{G}_{4 j}^{*}-\tilde{p}_{a} \tilde{G}_{4 j, n}^{*}\right) \mathrm{d} \Gamma \\
& \int_{\Omega}\left(\tilde{u}_{i} \tilde{B}_{i m}^{*}\right) \tilde{G}_{m j}^{*} \mathrm{~d} \Omega=0
\end{aligned}
$$


in which

$$
\tilde{\mathbf{B}}^{*}=\left[\begin{array}{ccc}
\left(\mu \Delta-\rho s^{2}\right) \delta_{\alpha \beta}+(\lambda+\mu) \partial_{\alpha} \partial_{\beta} & s \theta_{1} \partial_{\alpha} & s \theta_{2} \partial_{\alpha} \\
-F_{s} \partial_{\beta} & k_{w} \Delta+C_{w w} s & C_{w a} s \\
-\left(1-F_{s}\right) \partial_{\beta} & C_{w a} s & k_{a} \Delta+C_{a a} s
\end{array}\right]
$$

By substituting Eq (64) into (68) and using the property of Dirac's delta function $\delta(x-\xi)$, we arrive at the transformed dynamic unsaturated poroelastic boundary integral representation for the transformed internal displacements and pressures given in matrix form, i.e.,

$$
\begin{aligned}
& \left.c(\xi) \mathbf{I} \mid \begin{array}{l}
u_{\alpha}(\xi ; s) \\
p_{w}(\xi ; s) \\
p_{a}(\xi ; s)
\end{array}\right]=\int_{\Gamma}\left[\begin{array}{lll}
\tilde{U}_{\beta}^{W}(x, \xi ; s) & -\tilde{P}^{w W}(x, \xi ; s) & -\tilde{P}^{a W}(x, \xi ; s) \\
\tilde{U}_{\beta}^{A}(x, \xi ; s) & -\tilde{P}^{w A}(x, \xi ; s) & -\tilde{P}^{g A}(x, \xi ; s)
\end{array}\right]\left|\tilde{q}_{w}(x ; s)\right| d \Gamma \\
& -f_{\Gamma}\left[\begin{array}{lll}
\tilde{T}_{\alpha \beta}^{s}(x, \xi ; s) & \tilde{Q}_{\alpha}^{w S}(x, \xi ; s) & \tilde{Q}_{\alpha}^{a S}(x, \xi ; s) \\
\tilde{T}_{\beta}^{W}(x, \xi ; s) & \tilde{Q}^{w W}(x, \xi ; s) & \tilde{Q}^{a W}(x, \xi ; s) \\
\tilde{T}_{\beta}^{A}(x, \xi ; s) & \tilde{Q}^{w A}(x, \xi ; s) & \tilde{Q}^{a A}(x, \xi ; s)
\end{array}\right]\left|\begin{array}{c}
\tilde{p}_{\alpha}(x ; s) \\
\tilde{p}_{w}(x ; s)
\end{array}\right| d \Gamma
\end{aligned}
$$

Where the traction vector, the normal water flux and the normal gas flux are respectively

$$
\begin{gathered}
\tilde{t}_{\alpha}=\sigma_{\alpha \beta} n_{\beta}=\left[\left(\lambda \tilde{u}_{k, k}-F_{s}\left(\tilde{p}_{a}-\tilde{p}_{w}\right)+\tilde{p}_{a}\right) \delta_{\alpha \beta}+\mu\left(\tilde{u}_{\beta, \alpha}+\tilde{u}_{\alpha, \beta}\right)\right] n_{\beta} \\
\tilde{q}_{w}=-k_{w}\left(\tilde{p}_{w, n}+\rho_{w} s^{2} \tilde{u}_{\alpha} n_{\alpha}\right) \\
\tilde{q}_{a}=-k_{a}\left(\tilde{p}_{a, n}+\rho_{a} s^{2} \tilde{u}_{\alpha} n_{\alpha}\right)
\end{gathered}
$$

The coefficient $c_{i j}$ has a value $\delta_{i j}$ for points inside $\Omega$ and zero outside $\Omega$. The value of $c_{i j}$ for points on the boundary $\Gamma$ is determined from the Cauchy principal value of the integrals. It is equal to $0.5 \delta_{i j}$ for points on $\Gamma$ where the boundary is smooth.

Also the $\mathbf{T}^{s}, \tilde{\mathbf{Q}}^{w s}$ and $\tilde{\mathbf{Q}}^{a s}$ in Eq (70) can be interpreted as the adjoint terms to the traction vector $\tilde{t}_{\alpha}$, the water flux $\tilde{q}_{w}$ and the air flux $\tilde{q}_{a}$ as 


$$
\begin{gathered}
T_{\alpha \beta}^{S}=\left[\left(\lambda \tilde{U}_{k \beta, k}^{S}+s S_{w} \tilde{P}_{\beta}^{w S}+s S_{a} \tilde{P}_{\beta}^{a S}\right) \delta_{\alpha l}+\mu\left(\tilde{U}_{\alpha \beta, l}^{S}+\tilde{U}_{l \beta, \alpha}^{S}\right)\right] n_{l} \\
T_{\alpha}^{W}=\left[\left(\lambda \tilde{U}_{k, k}^{W}+s S_{w} \tilde{P}^{w W}+s S_{a} \tilde{P}^{a W}\right) \delta_{\alpha l}+\mu\left(\tilde{U}_{\alpha, l}^{W}+\tilde{U}_{l, \alpha}^{W}\right)\right] n_{l} \\
T_{\alpha}^{A}=\left[\left(\lambda \tilde{U}_{k, k}^{A}+s S_{w} \tilde{P}^{w A}+s S_{a} \tilde{P}^{a A}\right) \delta_{\alpha l}+\mu\left(\tilde{U}_{\alpha, l}^{A}+\tilde{U}_{l, \alpha}^{A}\right)\right] n_{l} \\
\tilde{Q}_{\alpha}^{w S}=k_{w} P_{\alpha, n}^{w S} \\
\tilde{Q}^{w W}=k_{w} P_{, n}^{w W} \\
\tilde{Q}^{w A}=k_{w} P_{, n}^{w A} \\
\tilde{Q}_{\alpha}^{a S}=k_{a} P_{\alpha, n}^{a S} \\
\tilde{Q}^{a W}=k_{a} P_{, n}^{a W} \\
\tilde{Q}^{a A}=k_{a} P_{, n}^{a A}
\end{gathered}
$$

Eq (70) can be compacted in index notation for the 2-D case as following

$$
c(\xi) \mathbf{I} \tilde{u}_{j}(\xi ; s)=\int_{\Gamma}\left(\tilde{G}_{i j}(x, \xi ; s) \tilde{t}_{i}(x ; s)-\tilde{F}_{i j}(x, \xi ; s) \tilde{u}_{i}(x ; s)\right) d \Gamma
$$

Where $\tilde{t}_{i}=\left[\begin{array}{lll}\tilde{t}_{\alpha} & \tilde{q}_{w} & \tilde{q}_{a}\end{array}\right]^{T}, \tilde{u}_{i}=\left[\begin{array}{lll}\tilde{u}_{\alpha} & \tilde{p}_{w} & \tilde{p}_{a}\end{array}\right]^{T}$ and also

$$
\begin{array}{r}
\tilde{G}_{i j}=\left[\begin{array}{ccc}
\tilde{U}_{\alpha \beta}^{S} & -\tilde{P}_{\alpha}^{w S} & -\tilde{P}_{\alpha}^{a S} \\
\tilde{U}_{\beta}^{W} & -\tilde{P}^{w W} & -\tilde{P}^{a W} \\
\tilde{U}_{\beta}^{A} & -\tilde{P}^{w A} & -\tilde{P}^{a A}
\end{array}\right] \\
\tilde{F}_{i j}=\left[\begin{array}{ccc}
\tilde{T}_{\alpha \beta}^{S} & \tilde{Q}_{\alpha}^{w S} & \tilde{Q}_{\alpha}^{a S} \\
\tilde{T}_{\beta}^{W} & \tilde{Q}^{w W} & \tilde{Q}^{a W} \\
\tilde{T}_{\beta}^{A} & \tilde{Q}^{w A} & \tilde{Q}^{a A}
\end{array}\right]
\end{array}
$$

With $i, j$ varies from one to four and $\alpha, \beta, k$ varies from one to two. 
The time dependent boundary integral equation for the unsaturated soil is obtained by a transformation to time domain.

$$
c(\xi) \mathbf{I} u_{j}(\xi ; t)=\int_{0}^{t} \int_{\Gamma}\left(G_{i j}(t-\tau ; x, \xi,) t_{i}(\tau ; x)-F_{i j}(t-\tau ; x, \xi) u_{i}(\tau ; x)\right) d \Gamma
$$

\section{Fundamental Solutions}

Here, the fundamental solution for the unsaturated poroelastic governing equation (64) is derived in Laplace transform domain. These solutions can be used in a time-dependent convolution quadrature-based BE formulation which needs only Laplace transformed fundamental solutions.

The physical interpretation of fundamental solution or kernel of a differential equation is a potential function $\Pi(x, \xi)$ or on the other hand, the response of the medium in the point $x$ to a point excitation $e(\xi)$ in a domain with infinite boundaries which is a Dirac delta function in space, i.e. $\delta(\xi)$ and either a Dirac delta function or a Heaviside step function i.e. $H(t)$ in time. Mathematically spoken a fundamental solution is a solution of the equation $\mathbf{B G}+\mathbf{I} \delta(x-y) \delta(t-\tau)=\mathbf{0}$ where the matrix of fundamental solutions is denoted by $\mathbf{G}$, the identity matrix by $\mathbf{I}$ and the matrix differential operator by $\mathbf{B}$.

In this study, because the operator type of the governing equations is an elliptical operator the explicit 2D Laplace transform domain fundamental solution can be derived by using the method of Kupradze et al. [34] or Hörmander [29].

The idea of this method is to reduce the highly complicated operator given in (63) to simple well known operators. An overview of this method is found in the original work by Hörmander [29] and more exemplary in References [38, 24, 16, 17, 18, 19, 20, 30, 32].

In this method, in the Laplace transform domain, the first stage is to find the matrix of cofactors $\tilde{\mathbf{B}}^{\text {co }}$ to calculate the inverse matrix of $\tilde{\mathbf{B}}$

$$
\tilde{\mathbf{B}} \tilde{\mathbf{B}}^{-1}=\mathbf{I} \text { with } \tilde{\mathbf{B}}^{-1}=\tilde{\mathbf{B}}^{\text {co }} / \operatorname{det}(\tilde{\mathbf{B}})
$$

For the second stage, we assume that $\varphi$ is a scalar solution to the equation 


$$
\operatorname{det}(\tilde{\mathbf{B}}) \mathbf{I} \varphi+\mathbf{I} \delta(x-\xi)=\mathbf{0} \quad \leftrightarrow \quad \operatorname{det}(\tilde{\mathbf{B}}) \varphi+\delta(x-\xi)=0
$$

Which gives

$$
\tilde{\mathbf{B}} \tilde{\mathbf{B}}^{\mathrm{co}} \varphi+\mathbf{I} \delta(x-\xi)=\mathbf{0}
$$

Consequently, we get

$$
\tilde{\mathbf{G}}=\tilde{\mathbf{B}}^{\mathrm{co}} \varphi
$$

It is known that the fundamental solution should satisfy the adjoint operator [49]. As shown before all the operators in (63) are elliptic and not self-adjoint. Therefore, for the deduction of fundamental solutions the adjoint operator $\tilde{\mathbf{B}}^{*}$ has to be used:

$$
\tilde{\mathbf{B}}^{*}=\left[\begin{array}{ccc}
\left(a_{1} \Delta+a_{3} s^{2}\right) \delta_{\alpha \beta}+a_{2} \partial_{\alpha} \partial_{\beta} & \left(s a_{4}+a_{5} s^{2}\right) \partial_{\alpha} & \left(s a_{6}+a_{7} s^{2}\right) \partial_{\alpha} \\
a_{8} \partial_{\beta} & a_{9} \Delta+a_{10} s & a_{11} s \\
a_{12} \partial_{\beta} & a_{11} s & a_{13} \Delta+a_{14} s
\end{array}\right]
$$

Where $a_{1}=\mu, a_{2}=\lambda+\mu, a_{3}=-\rho, a_{4}=S_{w}, a_{5}=-\rho_{w} . k_{w}, a_{6}=S_{a}, a_{7}=-\rho_{a} k_{a}, a_{8}=-F_{s}$, $a_{9}=k_{w}, a_{10}=C_{w w}, a_{11}=C_{w a}, a_{12}=-\left(1-F_{s}\right), a_{13}=k_{a}$ and $a_{14}=C_{a a}$.

At first following formula (78), the determinant of the operator $\tilde{\mathbf{B}}^{*}$ is calculated. This yields to the results

$$
\operatorname{det}\left(\tilde{\mathbf{B}}^{*}\right)=D_{1}\left(\Delta^{4}+\left(\frac{D_{2}}{D_{1}} s^{2}+\frac{D_{3}}{D_{1}} s\right) \Delta^{3}+\left(\frac{D_{4}}{D_{1}} s^{4}+\frac{D_{5}}{D_{1}} s^{3}+\frac{D_{6}}{D_{1}} s^{2}\right) \Delta^{2}+\left(\frac{D_{7}}{D_{1}} s^{5}+\frac{D_{8}}{D_{1}} s^{4}\right) \Delta+\frac{D_{9}}{D_{1}} s^{6}\right)
$$

where $D_{1}, D_{2}, D_{3}$ and $D_{4}$ are constants including above $a_{z}(z=\overline{1,14})$ coefficients (Appendix A). 
We can rewrite the above expression (83) into the following factorized form [34]:

$$
\operatorname{det}\left(\tilde{\mathbf{B}}^{*}\right)=\mu(\lambda+2 \mu) k_{w} k_{a}\left(\Delta-\lambda_{1}^{2}\right)\left(\Delta-\lambda_{2}^{2}\right)\left(\Delta-\lambda_{3}^{2}\right)\left(\Delta-\lambda_{4}^{2}\right)
$$

In which the coefficients $\lambda_{1}^{2}, \lambda_{2}^{2}, \lambda_{3}^{2}$ and $\lambda_{4}^{2}$ are the roots of this polynomial of order four of $\Delta$, which one of its roots is the $\lambda_{1}^{2}=\rho s^{2} / \mu$ that is related to the shear wave velocity propagating through the medium. The remaining part of equation (84) is a polynomial of order three of $\Delta$, which have three roots as $\lambda_{2}^{2}, \lambda_{3}^{2}, \lambda_{4}^{2}$ and they must be determined as these which satisfy

$$
\begin{gathered}
\lambda_{2}^{2}+\lambda_{3}^{2}+\lambda_{4}^{2}=\frac{\rho s^{2}}{(\lambda+2 \mu)}+\frac{F_{s} \rho_{w} s^{2}}{(\lambda+2 \mu)}+\frac{\rho_{a}\left(1-F_{s}\right) s^{2}}{(\lambda+2 \mu)} \\
-\frac{C_{a a} s}{k_{a}}-\frac{C_{w w} s}{k_{w}}-\frac{S_{w} F_{s} s}{(\lambda+2 \mu) k_{w}}-\frac{S_{a}\left(1-F_{s}\right) s}{(\lambda+2 \mu) k_{a}} \\
\lambda_{2}^{2} \lambda_{3}^{2}+\lambda_{2}^{2} \lambda_{4}^{2}+\lambda_{3}^{2} \lambda_{4}^{2}=-\frac{\rho C_{a a} s^{3}}{(\lambda+2 \mu) k_{a}}-\frac{\rho C_{w w} s^{3}}{(\lambda+2 \mu) k_{w}}-\frac{\rho_{w}\left(F_{s} C_{a a}-\left(1-F_{s}\right) C_{w a}\right) s^{3}}{(\lambda+2 \mu) k_{a}} \\
-\frac{\rho_{a}\left(-F_{s} C_{w a}+\left(1-F_{s}\right) C_{w w}\right) s^{3}}{(\lambda+2 \mu) k_{w}}+\frac{\left(C_{w w} C_{a a}-C_{w a}^{2}\right) s^{2}}{k_{w} k_{a}} \\
+\frac{S_{w}\left(F_{s} C_{a a}-C_{w a}\left(1-F_{s}\right)\right) s^{2}}{(\lambda+2 \mu) k_{w} k_{a}}+\frac{S_{a}\left(-F_{s} C_{w a}+C_{w w}\left(1-F_{s}\right)\right) s^{2}}{(\lambda+2 \mu) k_{w} k_{a}} \\
\lambda_{2}^{2} \lambda_{3}^{2} \lambda_{4}^{2}=\frac{\rho\left(C_{w w} C_{a a}-C_{w a}^{2}\right) s^{4}}{(\lambda+2 \mu) k_{w} k_{a}}
\end{gathered}
$$

These three roots correspond to the three compressional waves which are affected by the degree of saturation and the spatial distribution of fluids within the medium.

Biot demonstrate the existence of two kinds of compressional waves in a fully saturated medium: the fast wave for which the solid and fluid displacements are in phase, and the slow wave for which the displacements are out of phase. However, when the pore space is filled with two immiscible fluids, capillary forces are important and the existence of a third compressional wave (i.e., a second slow wave) is predicted in the medium [7]. 
From the relations (85) the coefficients $\lambda_{2}^{2}, \lambda_{3}^{2}, \lambda_{4}^{2}$ can be determined as the roots of the following cubic equation of unknown $\lambda^{2}$

$$
\left(\lambda^{2}\right)^{3}+\left[-\left(\lambda_{2}^{2}+\lambda_{3}^{2}+\lambda_{4}^{2}\right)\right]\left(\lambda^{2}\right)^{2}+\left[\left(\lambda_{2}^{2} \lambda_{3}^{2}+\lambda_{2}^{2} \lambda_{4}^{2}+\lambda_{3}^{2} \lambda_{4}^{2}\right)\right]\left(\lambda^{2}\right)+\left[-\left(\lambda_{2}^{2} \lambda_{3}^{2} \lambda_{4}^{2}\right)\right]=0
$$

Then, by introducing equation (84) into equation (78) the matrix of cofactors $\tilde{\mathbf{B}}^{* \text { co }}$ is obtained. The elements of this operator are presented in Appendix B.

Secondly, the scalar equation corresponding to (79) becomes

$$
\left(\Delta-\lambda_{1}^{2}\right)\left(\Delta-\lambda_{2}^{2}\right)\left(\Delta-\lambda_{3}^{2}\right)\left(\Delta-\lambda_{4}^{2}\right) \Phi+\delta(x-\xi)=0
$$

in which $\Phi$ is an interim operator, i.e.

$$
\Phi=\mu(\lambda+2 \mu) k_{w} k_{a} \varphi
$$

Equation (88) can be expressed as either of three Eqs (89), (90), (91) and (92):

$$
\begin{aligned}
& \left(\Delta-\lambda_{1}^{2}\right) \varphi_{1}+\delta(x-\xi)=0 \\
& \varphi_{1}=\left(\Delta-\lambda_{2}^{2}\right)\left(\Delta-\lambda_{3}^{2}\right)\left(\Delta-\lambda_{4}^{2}\right) \Phi \\
& \left(\Delta-\lambda_{2}^{2}\right) \varphi_{2}+\delta(x-\xi)=0 \\
& \varphi_{2}=\left(\Delta-\lambda_{1}^{2}\right)\left(\Delta-\lambda_{3}^{2}\right)\left(\Delta-\lambda_{4}^{2}\right) \Phi \\
& \left(\Delta-\lambda_{3}^{2}\right) \varphi_{3}+\delta(x-\xi)=0 \\
& \varphi_{3}=\left(\Delta-\lambda_{1}^{2}\right)\left(\Delta-\lambda_{2}^{2}\right)\left(\Delta-\lambda_{4}^{2}\right) \Phi \\
& \left(\Delta-\lambda_{4}^{2}\right) \varphi_{4}+\delta(x-\xi)=0 \\
& \varphi_{4}=\left(\Delta-\lambda_{1}^{2}\right)\left(\Delta-\lambda_{2}^{2}\right)\left(\Delta-\lambda_{3}^{2}\right) \Phi
\end{aligned}
$$


The above differential equations are of the familiar Helmholtz type. The fundamental solution of Helmholtz differential equations for an only $r$-dependent fully symmetric two-dimensional domain is [2]:

$$
\varphi_{i}=\frac{K_{0}\left(\lambda_{i} r\right)}{2 \pi}, \quad i=1,2,3,4
$$

By definition of $\varphi_{1}, \varphi_{2}$ and $\varphi_{3}$, it is deduced

$$
\begin{aligned}
& \frac{1}{\lambda_{2}^{2}-\lambda_{1}^{2}}\left[\frac{\varphi_{3}-\varphi_{2}}{\lambda_{3}^{2}-\lambda_{2}^{2}}-\frac{\varphi_{3}-\varphi_{1}}{\lambda_{3}^{2}-\lambda_{1}^{2}}\right]=\left(\Delta-\lambda_{4}^{2}\right) \Phi \\
& \frac{1}{\lambda_{2}^{2}-\lambda_{1}^{2}}\left[\frac{\varphi_{4}-\varphi_{1}}{\lambda_{4}^{2}-\lambda_{1}^{2}}-\frac{\varphi_{4}-\varphi_{2}}{\lambda_{4}^{2}-\lambda_{2}^{2}}\right]=-\left(\Delta-\lambda_{3}^{2}\right) \Phi
\end{aligned}
$$

Then,

$$
\Phi=\frac{1}{\left(\lambda_{3}^{2}-\lambda_{4}^{2}\right)\left(\lambda_{2}^{2}-\lambda_{1}^{2}\right)}\left[\frac{\varphi_{3}-\varphi_{2}}{\lambda_{3}^{2}-\lambda_{2}^{2}}-\frac{\varphi_{3}-\varphi_{1}}{\lambda_{3}^{2}-\lambda_{1}^{2}}+\frac{\varphi_{4}-\varphi_{1}}{\lambda_{4}^{2}-\lambda_{1}^{2}}-\frac{\varphi_{4}-\varphi_{2}}{\lambda_{4}^{2}-\lambda_{2}^{2}}\right]
$$

Replacing equation (93) into (96), it is obtained

$$
\varphi=\frac{1}{2 \pi \mu(\lambda+2 \mu) k_{w} k_{a}}\left\{\begin{array}{l}
\frac{K_{0}\left(\lambda_{1} r\right)}{\left(\lambda_{1}^{2}-\lambda_{3}^{2}\right)\left(\lambda_{1}^{2}-\lambda_{4}^{2}\right)\left(\lambda_{1}^{2}-\lambda_{2}^{2}\right)}+\frac{K_{0}\left(\lambda_{2} r\right)}{\left(\lambda_{2}^{2}-\lambda_{4}^{2}\right)\left(\lambda_{2}^{2}-\lambda_{3}^{2}\right)\left(\lambda_{2}^{2}-\lambda_{1}^{2}\right)}+ \\
\frac{K_{0}\left(\lambda_{3} r\right)}{\left(\lambda_{3}^{2}-\lambda_{2}^{2}\right)\left(\lambda_{3}^{2}-\lambda_{1}^{2}\right)\left(\lambda_{3}^{2}-\lambda_{4}^{2}\right)}+\frac{K_{0}\left(\lambda_{4} r\right)}{\left(\lambda_{4}^{2}-\lambda_{1}^{2}\right)\left(\lambda_{4}^{2}-\lambda_{2}^{2}\right)\left(\lambda_{4}^{2}-\lambda_{3}^{2}\right)}
\end{array}\right\}
$$

In which $K_{0}\left(\lambda_{i} r\right)$ is the modified Bessel function of the second kind of order zero with the argument $r=|x-\xi|$ which denoted the distance between a load point and an observation point. After all, we can determine the components of fundamental solution tensor by applying the matrix of cofactors $\tilde{\mathbf{B}}^{* \text { co }}$ to the scalar function $\varphi$ which are:

\section{Displacement caused by a Dirac force in the solid.}


(98)

$$
\begin{aligned}
& \tilde{G}_{\alpha \beta}^{*}=\tilde{U}_{\alpha \beta}^{s}=\left\{\begin{array}{l}
\frac{-(\lambda+\mu) \Lambda^{2}}{\rho s^{2}} \frac{\left(\lambda_{1}^{2}-K_{s s 1}^{2}\right)\left(\lambda_{1}^{2}-K_{s s 2}^{2}\right)}{\left(\lambda_{1}^{2}-\lambda_{2}^{2}\right)\left(\lambda_{1}^{2}-\lambda_{3}^{2}\right)\left(\lambda_{1}^{2}-\lambda_{4}^{2}\right)}\left(R_{1} \lambda_{1} K_{1}\left(\lambda_{1} r\right)+R_{2} \lambda_{1}^{2} K_{0}\left(\lambda_{1} r\right)\right)+ \\
\frac{-(\lambda+\mu) \Lambda^{2}}{\rho s^{2}} \frac{\left(\lambda_{2}^{2}-K_{s s 1}^{2}\right)\left(\lambda_{2}^{2}-K_{s s 2}^{2}\right)}{\left(\lambda_{2}^{2}-\lambda_{1}^{2}\right)\left(\lambda_{2}^{2}-\lambda_{4}^{2}\right)\left(\lambda_{2}^{2}-\lambda_{3}^{2}\right)}\left(R_{1} \lambda_{2} K_{1}\left(\lambda_{2} r\right)+R_{2} \lambda_{2}^{2} K_{0}\left(\lambda_{2} r\right)\right)+ \\
\left.\frac{-(\lambda+\mu) \Lambda^{2}}{\rho s^{2}} \frac{\left(\lambda_{3}^{2}-K_{s s 1}^{2}\right)\left(\lambda_{3}^{2}-K_{s s 2}^{2}\right)}{\left(\lambda_{3}^{2}-\lambda_{4}^{2}\right)\left(\lambda_{3}^{2}-\lambda_{2}^{2}\right)\left(\lambda_{3}^{2}-\lambda_{1}^{2}\right)}\left(R_{1} \lambda_{3} K_{1}\left(\lambda_{3} r\right)+R_{2} \lambda_{3}^{2} K_{0}\left(\lambda_{3} r\right)\right)+\right\} \\
\frac{-(\lambda+\mu) \Lambda^{2}}{\rho s^{2}} \frac{\left(\lambda_{4}^{2}-K_{s s 1}^{2}\right)\left(\lambda_{4}^{2}-K_{s s 2}^{2}\right)}{\left(\lambda_{4}^{2}-\lambda_{3}^{2}\right)\left(\lambda_{4}^{2}-\lambda_{1}^{2}\right)\left(\lambda_{4}^{2}-\lambda_{2}^{2}\right)}\left(R_{1} \lambda_{4} K_{1}\left(\lambda_{4} r\right)+R_{2} \lambda_{4}^{2} K_{0}\left(\lambda_{4} r\right)\right)
\end{array}\right\} \\
&+\frac{\delta_{\alpha \beta}}{2 \pi \mu} K_{0}\left(\lambda_{1} r\right)
\end{aligned}
$$

with

$$
\begin{aligned}
& R_{1}=\left(\frac{2 x_{\alpha} x_{\beta}}{r^{3}}-\frac{\delta_{\alpha \beta}}{r}\right), R_{2}=\frac{x_{\alpha} x_{\beta}}{r^{2}}, \Lambda^{2}=\frac{\rho}{(\lambda+2 \mu)} s^{2} \text { and } \\
& K_{s s 1}^{2}+K_{s s 2}^{2}=\frac{-S_{w} F_{s} s}{(\lambda+\mu) k_{w}}+\frac{-S_{a}\left(1-F_{s}\right) s}{(\lambda+\mu) k_{a}}-\frac{\left(k_{w} C_{a a}+C_{w w} k_{a}\right) s}{k_{w} k_{a}}+\frac{\rho_{w} k_{w} F_{s} s^{2}}{(\lambda+\mu) k_{w}}+\frac{\rho_{a} k_{a}\left(1-F_{s}\right) s^{2}}{(\lambda+\mu) k_{a}} \\
& K_{s s 1}^{2} K_{s s 2}^{2}=\left\{\begin{array}{l}
\frac{\left(-C_{w a}^{2}+C_{w w} C_{a a}\right)}{k_{w} k_{a}}+\frac{S_{w}\left(-C_{w a}\left(1-F_{s}\right)+F_{s} C_{a a}\right)}{(\lambda+\mu) k_{w} k_{a}}+\frac{S_{a}\left(-F_{s} C_{w a}+C_{w w}\left(1-F_{s}\right)\right)}{(\lambda+\mu) k_{w} k_{a}} \\
+\frac{-\rho_{w} k_{w}\left(-C_{w a}\left(1-F_{s}\right)+F_{s} C_{a a}\right) s}{(\lambda+\mu) k_{w} k_{a}}+\frac{-\rho_{a} k_{a}\left(-F_{s} C_{w a}+C_{w w}\left(1-F_{s}\right)\right) s}{(\lambda+\mu) k_{w} k_{a}}
\end{array} \mid s^{2}\right.
\end{aligned}
$$

Water pressure caused by a Dirac force in the solid.

(99) 


$$
\begin{aligned}
& \tilde{G}_{3 \beta}^{*}=\tilde{P}_{\beta}^{w S}= \\
& \frac{F_{s}}{2 \pi(\lambda+2 \mu) k_{w}} \frac{x_{\beta}}{r}\left\{\begin{array}{l}
\frac{-\lambda_{2}}{\left(\lambda_{4}^{2}-\lambda_{2}^{2}\right)\left(\lambda_{3}^{2}-\lambda_{2}^{2}\right)}\left(\lambda_{2}^{2}-\frac{\left(C_{w a}\left(1-F_{s}\right)-F_{s} C_{a a}\right)}{F_{s} k_{a}} s\right) K_{1}\left(\lambda_{2} r\right) \\
\left.+\frac{-\lambda_{3}}{\left(\lambda_{4}^{2}-\lambda_{3}^{2}\right)\left(\lambda_{2}^{2}-\lambda_{3}^{2}\right)}\left(\lambda_{3}^{2}-\frac{\left(C_{w a}\left(1-F_{s}\right)-F_{s} C_{a a}\right)}{F_{s} k_{a}} s\right) K_{1}\left(\lambda_{3} r\right)\right\} \\
\left.+\frac{-\lambda_{4}}{\left(\lambda_{3}^{2}-\lambda_{4}^{2}\right)\left(\lambda_{2}^{2}-\lambda_{4}^{2}\right)}\left(\lambda_{4}^{2}-\frac{\left(C_{w a}\left(1-F_{s}\right)-F_{s} C_{a a}\right)}{F_{s} k_{a}} s\right) K_{1}\left(\lambda_{4} r\right)\right\}
\end{array}\right\}
\end{aligned}
$$

Air pressure caused by a Dirac force in the solid.

$$
\begin{aligned}
& \tilde{G}_{4 \beta}^{*}=\tilde{P}_{\beta}^{a S}=
\end{aligned}
$$

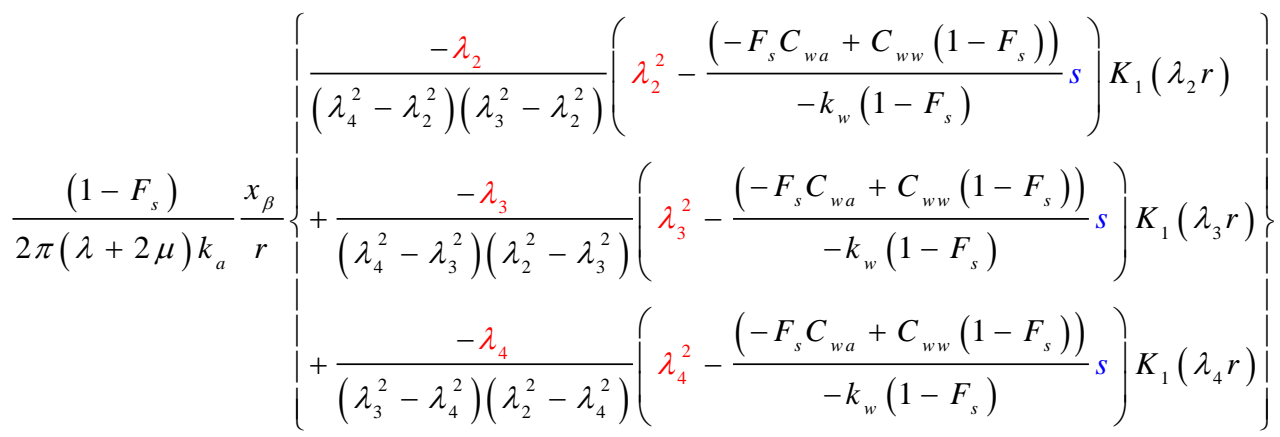

$$
\begin{aligned}
& \tilde{G}_{\alpha 3}^{*}=\tilde{U}_{\alpha}^{W}=
\end{aligned}
$$

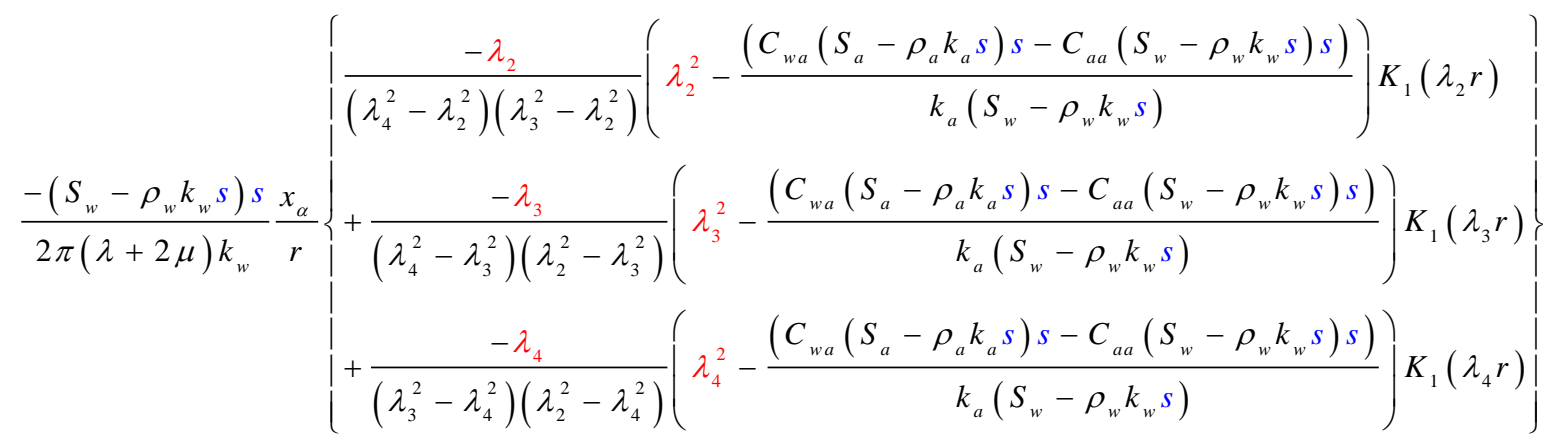


Water pressure caused by a Dirac source in the water fluid.

$$
\begin{aligned}
\tilde{G}_{33}^{*}=\tilde{P}^{w W}= \\
\frac{1}{2 \pi k_{w}}\left\{\begin{array}{l}
\left.\frac{1}{\left(\lambda_{4}^{2}-\lambda_{2}^{2}\right)\left(\lambda_{3}^{2}-\lambda_{2}^{2}\right)}\left(\lambda_{2}^{2}-K_{w}^{2}\right)\left(\lambda_{2}^{2}-\Lambda_{w}^{2}\right) K_{0}\left(\lambda_{2} r\right)+\right\} \\
\left.\frac{1}{\left(\lambda_{4}^{2}-\lambda_{3}^{2}\right)\left(\lambda_{2}^{2}-\lambda_{3}^{2}\right)}\left(\lambda_{3}^{2}-K_{w}^{2}\right)\left(\lambda_{3}^{2}-\Lambda_{w}^{2}\right) K_{0}\left(\lambda_{3} r\right)+\right\} \\
\frac{1}{\left(\lambda_{3}^{2}-\lambda_{4}^{2}\right)\left(\lambda_{2}^{2}-\lambda_{4}^{2}\right)}\left(\lambda_{4}^{2}-K_{w}^{2}\right)\left(\lambda_{4}^{2}-\Lambda_{w}^{2}\right) K_{0}\left(\lambda_{4} r\right)
\end{array}\right]
\end{aligned}
$$

With

$$
\begin{gathered}
\lambda_{1}^{2}=\frac{\rho}{\mu} s^{2}, K_{w}^{2} \Lambda_{w}^{2}=\frac{-\rho C_{a a} s^{3}}{(\lambda+2 \mu) k_{a}} \text { and } \\
K_{w}^{2}+\Lambda_{w}^{2}=\frac{-S_{a}\left(1-F_{s}\right) s}{(\lambda+2 \mu) k_{a}}-\frac{C_{a a} s}{k_{a}}+\frac{\rho_{a} k_{a}\left(1-F_{s}\right) s^{2}}{(\lambda+2 \mu) k_{a}}+\frac{\rho s^{2}}{(\lambda+2 \mu)}
\end{gathered}
$$

Air pressure caused by a Dirac source in the water fluid.

$$
\begin{aligned}
& \tilde{G}_{43}^{*}=\tilde{P}^{a W}= \\
& \frac{s}{2 \pi(\lambda+2 \mu) k_{w} k_{a}}\left\{\begin{array}{l}
\frac{\left(-(\lambda+2 \mu) C_{w a}+\left(\rho_{w} k_{w} s-S_{w}\right)\left(1-F_{s}\right)\right) \lambda_{2}^{2}+\rho C_{w a} s^{2}}{\left(\lambda_{4}^{2}-\lambda_{2}^{2}\right)\left(\lambda_{3}^{2}-\lambda_{2}^{2}\right)} K_{0}\left(\lambda_{2} r\right) \\
\left(\lambda_{4}^{2}-\lambda_{3}^{2}\right)\left(\lambda_{2}^{2}-\lambda_{3}^{2}\right) \\
+\frac{\left(-(\lambda+2 \mu) C_{w a}+\left(\rho_{w} k_{w} s-S_{w}\right)\left(1-F_{s}\right)\right) \lambda_{3}^{2}+\rho C_{w a} s^{2}}{\left(\lambda_{3}^{2}-\lambda_{4}^{2}\right)\left(\lambda_{2}^{2}-\lambda_{4}^{2}\right)} C_{0}\left(\lambda_{3} r\right)
\end{array}\right\}
\end{aligned}
$$

Displacement caused by a Dirac source in the air fluid. 


$$
\begin{aligned}
& \tilde{G}_{\alpha 4}^{*}=\tilde{U}_{\alpha}^{A}=
\end{aligned}
$$

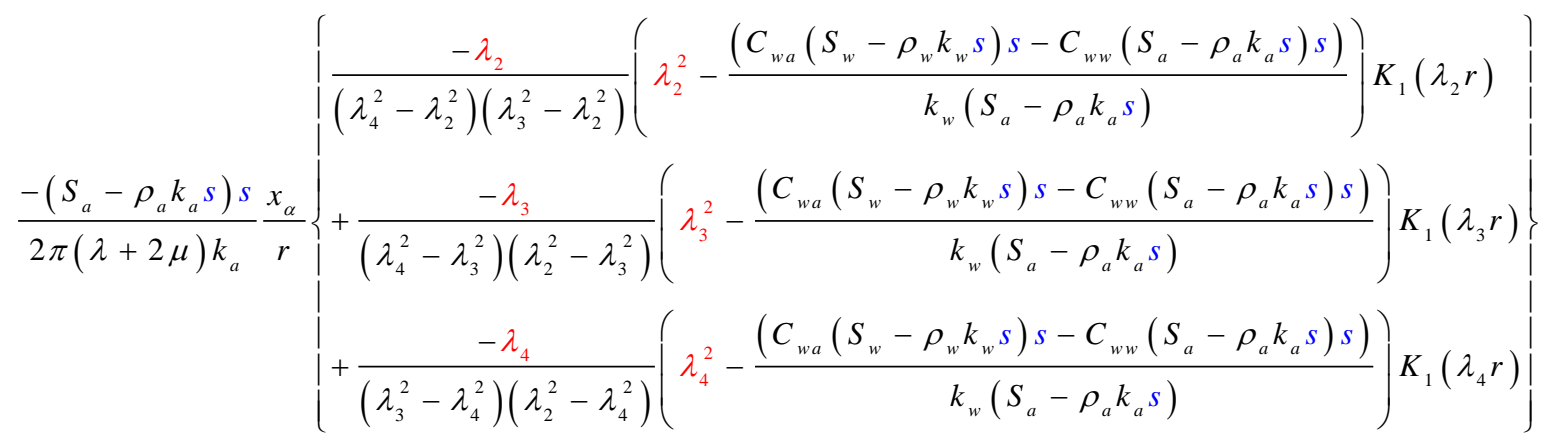

with

$$
\lambda_{1}^{2}=\frac{\rho}{\mu} s^{2} \text { and } K_{s a}^{2}=\frac{\left(C_{w a}\left(S_{w}-\rho_{w} k_{w} s\right) s-C_{w w}\left(S_{a}-\rho_{a} k_{a} s\right) s\right)}{k_{w}\left(S_{a}-\rho_{a} k_{a} s\right)}
$$

Water pressure caused by a Dirac source in the air fluid.

$$
\begin{aligned}
& \tilde{G}_{34}^{*}=\tilde{P}^{w A}= \\
& \frac{s}{2 \pi(\lambda+2 \mu) k_{w} k_{a}}\left\{\begin{array}{l}
\frac{\left(-(\lambda+2 \mu) C_{w a}+\left(\rho_{a} k_{a} s-S_{a}\right) F_{s}\right) \lambda_{2}^{2}+\rho C_{w a} s^{2}}{\left(\lambda_{4}^{2}-\lambda_{2}^{2}\right)\left(\lambda_{3}^{2}-\lambda_{2}^{2}\right)} K_{0}\left(\lambda_{2} r\right) \\
\left(\lambda_{4}^{2}-\lambda_{3}^{2}\right)\left(\lambda_{2}^{2}-\lambda_{3}^{2}\right) \\
+\frac{\left(-(\lambda+2 \mu) C_{w a}+\left(\rho_{a} k_{a} s-S_{a}\right) F_{s}\right) \lambda_{3}^{2}+\rho C_{w a} s^{2}}{\left(\lambda_{3}^{2}-\lambda_{4}^{2}\right)\left(\lambda_{2}^{2}-\lambda_{4}^{2}\right)} K_{0}\left(\lambda_{3} r\right)
\end{array}\right\}
\end{aligned}
$$

Air pressure caused by a Dirac source in the air fluid.

(106) 


$$
\begin{aligned}
& \tilde{G}_{44}^{*}=\tilde{P}^{a A}= \\
& \left.\frac{1}{2 \pi k_{a}} \mid \begin{array}{l}
\frac{1}{\left(\lambda_{4}^{2}-\lambda_{2}^{2}\right)\left(\lambda_{3}^{2}-\lambda_{2}^{2}\right)}\left(\lambda_{2}^{2}-K_{a}^{2}\right)\left(\lambda_{2}^{2}-\Lambda_{a}^{2}\right) K_{0}\left(\lambda_{2} r\right) \\
+\frac{1}{\left(\lambda_{4}^{2}-\lambda_{3}^{2}\right)\left(\lambda_{2}^{2}-\lambda_{3}^{2}\right)}\left(\lambda_{3}^{2}-K_{a}^{2}\right)\left(\lambda_{3}^{2}-\Lambda_{a}^{2}\right) K_{0}\left(\lambda_{3} r\right) \\
+\frac{1}{\left(\lambda_{3}^{2}-\lambda_{4}^{2}\right)\left(\lambda_{2}^{2}-\lambda_{4}^{2}\right)}\left(\lambda_{4}^{2}-K_{a}^{2}\right)\left(\lambda_{4}^{2}-\Lambda_{a}^{2}\right) K_{0}\left(\lambda_{4} r\right)
\end{array}\right]
\end{aligned}
$$

With

$$
\begin{gathered}
\lambda_{1}^{2}=\frac{\rho}{\mu} s^{2}, K_{a}^{2} \Lambda_{a}^{2}=\frac{-\rho C_{w w} s^{3}}{(\lambda+2 \mu) k_{w}} \text { and } \\
K_{a}^{2}+\Lambda_{a}^{2}=\frac{-S_{w} F_{s} s}{(\lambda+2 \mu) k_{w}}-\frac{C_{w w} s}{k_{w}}+\frac{\rho_{w} k_{w} F_{s} s^{2}}{(\lambda+2 \mu) k_{w}}+\frac{\rho s^{2}}{(\lambda+2 \mu)}
\end{gathered}
$$

In the derivation of the multiphase poroelastodynamic boundary integral equation (70) several abbreviations corresponding to an 'adjoint' traction or flux are introduced (Eqs. 72, 73, 74). At first, the 'adjoint' traction solution is presented. However, due to the extensive only parts are given

$$
\left.\left(\tilde{U}_{\alpha \beta, l}^{s}+\tilde{U}_{l \beta, \alpha}^{s}\right) n_{l}=2 n_{l} \mid \begin{array}{l}
\left.C_{1}^{s s}\left(R_{3} \lambda_{1}^{2} K_{0}\left(\lambda_{1} r\right)+\left(\frac{2}{r} R_{3}-R_{4} \lambda_{1}^{2}\right) \lambda_{1} K_{1}\left(\lambda_{1} r\right)\right)+\right\} \\
C_{2}^{s s}\left(R_{3} \lambda_{2}^{2} K_{0}\left(\lambda_{2} r\right)+\left(\frac{2}{r} R_{3}-R_{4} \lambda_{2}^{2}\right) \lambda_{2} K_{1}\left(\lambda_{2} r\right)\right)+\mid \\
C_{3}^{s S}\left(R_{3} \lambda_{3}^{2} K_{0}\left(\lambda_{3} r\right)+\left(\frac{2}{r} R_{3}-R_{4} \lambda_{3}^{2}\right) \lambda_{3} K_{1}\left(\lambda_{3} r\right)\right)+ \\
\left.C_{4}^{s S}\left(R_{3} \lambda_{4}^{2} K_{0}\left(\lambda_{4} r\right)+\left(\frac{2}{r} R_{3}-R_{4} \lambda_{4}^{2}\right) \lambda_{4} K_{1}\left(\lambda_{4} r\right)\right)\right\}
\end{array}\right\}
$$

Where 


$$
\begin{aligned}
& R_{3}=\left(-4 r_{, \alpha} r_{, \beta} r_{, n}+r_{, \beta} n_{\alpha}+r_{, \alpha} n_{\beta}+r_{, n} \delta_{\alpha \beta}\right) / r \\
& R_{4}=r_{, n} r_{, \alpha} r_{, \beta} \\
& \tilde{U}_{k \beta, k}^{s} \delta_{\alpha l} n_{l}=-r_{, \beta} n_{\alpha}\left(C_{1}^{s S} \lambda_{1}^{3} K_{1}\left(\lambda_{1} r\right)+C_{2}^{s S} \lambda_{2}^{3} K_{1}\left(\lambda_{2} r\right)+C_{3}^{s S} \lambda_{3}^{3} K_{1}\left(\lambda_{3} r\right)+C_{4}^{s S} \lambda_{4}^{3} K_{1}\left(\lambda_{4} r\right)\right) \\
& -r_{, k} n_{\alpha} C_{5}^{s S} \lambda_{1} K_{1}\left(\lambda_{1} r\right) \\
& \left(\tilde{U}_{\alpha, l}^{W}+\tilde{U}_{l, \alpha}^{W}\right) n_{l}=2\left[\begin{array}{l}
\frac{n_{\alpha}-2 r_{, n} r_{, \alpha}}{r}\left(C_{1}^{s W} \lambda_{2} K_{1}\left(\lambda_{2} r\right)+C_{2}^{s W} \lambda_{3} K_{1}\left(\lambda_{3} r\right)+C_{3}^{s W} \lambda_{4} K_{1}\left(\lambda_{4} r\right)\right) \\
-r_{, n} r_{, \alpha}\left(C_{1}^{s W} \lambda_{2}^{2} K_{0}\left(\lambda_{2} r\right)+C_{2}^{s W} \lambda_{3}^{2} K_{0}\left(\lambda_{3} r\right)+C_{3}^{s W} \lambda_{4}^{2} K_{0}\left(\lambda_{4} r\right)\right)
\end{array}\right] \text { (108.a) } \\
& \tilde{U}_{k, k}^{W} \delta_{\alpha l} n_{l}=-n_{\alpha}\left(C_{1}^{s W} \lambda_{2}^{2} K_{0}\left(\lambda_{2} r\right)+C_{2}^{s W} \lambda_{3}^{2} K_{0}\left(\lambda_{3} r\right)+C_{3}^{s W} \lambda_{4}^{2} K_{0}\left(\lambda_{4} r\right)\right) \\
& \left(\tilde{U}_{\alpha, l}^{A}+\tilde{U}_{l, \alpha}^{A}\right) n_{l}=2\left[\begin{array}{l}
\frac{n_{\alpha}-2 r_{, n} r_{, \alpha}}{r}\left(C_{1}^{s A} \lambda_{2} K_{1}\left(\lambda_{2} r\right)+C_{2}^{s A} \lambda_{3} K_{1}\left(\lambda_{3} r\right)+C_{3}^{s A} \lambda_{4} K_{1}\left(\lambda_{4} r\right)\right) \\
-r_{, n} r_{, \alpha}\left(C_{1}^{s A} \lambda_{2}^{2} K_{0}\left(\lambda_{2} r\right)+C_{2}^{s A} \lambda_{3}^{2} K_{0}\left(\lambda_{3} r\right)+C_{3}^{s A} \lambda_{4}^{2} K_{0}\left(\lambda_{4} r\right)\right)
\end{array}\right] \\
& \tilde{U}_{k, k}^{A} \delta_{\alpha l} n_{l}=-n_{\alpha}\left(C_{1}^{s A} \lambda_{2}^{2} K_{0}\left(\lambda_{2} r\right)+C_{2}^{s A} \lambda_{3}^{2} K_{0}\left(\lambda_{3} r\right)+C_{3}^{s A} \lambda_{4}^{2} K_{0}\left(\lambda_{4} r\right)\right)
\end{aligned}
$$

The other explicit expressions are

$$
\begin{array}{r}
\tilde{Q}_{\alpha}^{w S}=\frac{n_{\alpha}-2 r_{, n} r_{, \alpha}}{r}\left(C_{1}^{w S} \lambda_{2} K_{1}\left(\lambda_{2} r\right)+C_{2}^{w S} \lambda_{3} K_{1}\left(\lambda_{3} r\right)+C_{3}^{w S} \lambda_{4} K_{1}\left(\lambda_{4} r\right)\right) \\
-r_{, n} r_{, \alpha}\left(C_{1}^{w S} \lambda_{2}^{2} K_{0}\left(\lambda_{2} r\right)+C_{2}^{w S} \lambda_{3}^{2} K_{0}\left(\lambda_{3} r\right)+C_{3}^{w S} \lambda_{4}^{2} K_{0}\left(\lambda_{4} r\right)\right) \\
\tilde{Q}^{w W}=-r_{, n}\left(C_{1}^{w W} \lambda_{2} K_{1}\left(\lambda_{2} r\right)+C_{2}^{w W} \lambda_{3} K_{1}\left(\lambda_{3} r\right)+C_{3}^{w W} \lambda_{4} K_{1}\left(\lambda_{4} r\right)\right) \\
\tilde{Q}^{w A}=-r_{, n}\left(C_{1}^{w A} \lambda_{2} K_{1}\left(\lambda_{2} r\right)+C_{2}^{w A} \lambda_{3} K_{1}\left(\lambda_{3} r\right)+C_{3}^{w A} \lambda_{4} K_{1}\left(\lambda_{4} r\right)\right) \\
\tilde{Q}_{\alpha}^{a S}=\frac{n_{\alpha}-2 r_{, n} r_{, \alpha}}{r}\left(C_{1}^{a S} \lambda_{2} K_{1}\left(\lambda_{2} r\right)+C_{2}^{a S} \lambda_{3} K_{1}\left(\lambda_{3} r\right)+C_{3}^{a S} \lambda_{4} K_{1}\left(\lambda_{4} r\right)\right) \\
\quad-r_{, n} r_{, \alpha}\left(C_{1}^{a S} \lambda_{2}^{2} K_{0}\left(\lambda_{2} r\right)+C_{2}^{a S} \lambda_{3}^{2} K_{0}\left(\lambda_{3} r\right)+C_{3}^{a S} \lambda_{4}^{2} K_{0}\left(\lambda_{4} r\right)\right)
\end{array}
$$




$$
\begin{gathered}
\tilde{Q}^{a W}=-r_{, n}\left(C_{1}^{a W} \lambda_{2} K_{1}\left(\lambda_{2} r\right)+C_{2}^{a W} \lambda_{3} K_{1}\left(\lambda_{3} r\right)+C_{3}^{a W} \lambda_{4} K_{1}\left(\lambda_{4} r\right)\right) \\
\tilde{Q}^{a A}=-r_{, n}\left(C_{1}^{a A} \lambda_{2} K_{1}\left(\lambda_{2} r\right)+C_{2}^{a A} \lambda_{3} K_{1}\left(\lambda_{3} r\right)+C_{3}^{a A} \lambda_{4} K_{1}\left(\lambda_{4} r\right)\right)
\end{gathered}
$$

In which the coefficients are presented in Appendix C.

\section{Singular behaviour}

As shown in part 3, the boundary integral equation is obtained by moving $\xi$ to the boundary $\Gamma$. Then in order to determine the unknown boundary data, it is necessary to know the behaviour of the fundamental solutions when $r=|\xi-x|$ tends to zero, i.e. when an integration point $x$ approaches a collocation point $\xi$. Simple series expansions of the fundamental solutions with respect to the variable $r=|\xi-x|$ shows that the singularity of these solutions in the limit $r \rightarrow 0$ is equal to the elastostatic, poro-elastostatic or the acoustic fundamental solutions (Table 1).

First consider $\tilde{G}_{33}^{*}=\tilde{P}^{w W}$, the pressure due to the Dirac source in the water fluid. As $r \rightarrow 0$, so does the argument of the modified Bessel functions. The limiting form of $K_{0}\left(\lambda_{i} r\right)$ as $r \rightarrow 0$ is

$$
K_{0}\left(\lambda_{i} r\right)=-\ln \left(\lambda_{i} r\right)=-\ln \left(\lambda_{i}\right)+\ln (1 / r)
$$

Thus the equation (102) reduces to

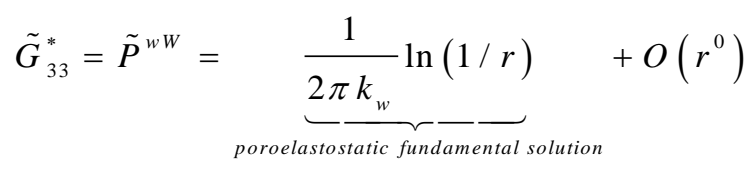

Similarly for $\tilde{G}_{44}^{*}=\tilde{P}^{a A}$, it is obtained 


$$
\tilde{G}_{44}^{*}=\tilde{P}^{a A}=\frac{1}{2 \pi k_{a}} \ln (1 / r)+O\left(r^{0}\right)
$$

Second consider $\tilde{G}_{\alpha 3}^{*}=\tilde{U}_{\alpha}^{W}$, Eq. (101) can rewritten as

$$
\begin{aligned}
\tilde{G}_{\alpha 3}^{*}=\tilde{U}_{\alpha}^{W}=\frac{s\left(S_{w}-\rho_{w} k_{w} s\right)}{4 \pi(\lambda+2 \mu) k_{w}} \frac{x_{\alpha}}{\left(\lambda_{4}^{2}-\lambda_{2}^{2}\right)\left(\lambda_{4}^{2}-\lambda_{3}^{2}\right)\left(\lambda_{3}^{2}-\lambda_{2}^{2}\right)} f(r)+ \\
\frac{s\left(S_{w}-\rho_{w} k_{w} s\right)}{4 \pi(\lambda+2 \mu) k_{w}} \frac{x_{\alpha}}{\left(\lambda_{4}^{2}-\lambda_{2}^{2}\right)\left(\lambda_{4}^{2}-\lambda_{3}^{2}\right)\left(\lambda_{3}^{2}-\lambda_{2}^{2}\right)}\left\{\begin{array}{l}
-\left(\lambda_{2}^{2}-M_{w}\right)\left(\lambda_{4}^{2}-\lambda_{3}^{2}\right) \lambda_{2}^{2} K_{0}\left(\lambda_{2} r\right) \\
\left.+\left(\lambda_{3}^{2}-M_{w}\right)\left(\lambda_{4}^{2}-\lambda_{2}^{2}\right) \lambda_{3}^{2} K_{0}\left(\lambda_{3} r\right)\right\} \\
\left.-\left(\lambda_{4}^{2}-M_{w}\right)\left(\lambda_{3}^{2}-\lambda_{2}^{2}\right) \lambda_{4}^{2} K_{0}\left(\lambda_{4} r\right)\right\}
\end{array}\right.
\end{aligned}
$$

Where

$$
\begin{gathered}
M_{w}=\frac{\left(C_{w a}\left(S_{a}-\rho_{a} k_{a} s\right) s-C_{a a}\left(S_{w}-\rho_{w} k_{w} s\right) s\right)}{k_{a}\left(S_{w}-\rho_{w} k_{w} s\right)} \\
f(r)=\frac{\left(\lambda_{2}^{2}-M_{w}\right)\left(\lambda_{4}^{2}-\lambda_{3}^{2}\right) \lambda_{2}^{2} r^{2} K_{2}\left(\lambda_{2} r\right)-\left(\lambda_{3}^{2}-M_{w}\right)\left(\lambda_{4}^{2}-\lambda_{2}^{2}\right) \lambda_{3}^{2} r^{2} K_{2}\left(\lambda_{3} r\right)+\left(\lambda_{4}^{2}-M_{w}\right)\left(\lambda_{3}^{2}-\lambda_{2}^{2}\right) \lambda_{4}^{2} r^{2} K_{2}\left(\lambda_{4} r\right)}{r^{2}}
\end{gathered}
$$

In which

$$
K_{2}\left(\lambda_{2} r\right)=K_{0}\left(\lambda_{2} r\right)+\frac{2}{\lambda_{2} r} K_{1}\left(\lambda_{2} r\right)
$$

Therefore it is of interest to find the value of $f(r)$ when $r \rightarrow 0$. Accordingly, we note that $K_{2}(x)=\frac{2}{x^{2}}$ as $x \rightarrow 0$. Thus the numerator and denominator of $f(r)$ tend to zero as $r \rightarrow 0$. This gives us the case 0 / 0 . We apply L'Hôpital's rule and note that $K_{1}(x)=\frac{1}{x}$ as $x \rightarrow 0$

$$
\lim _{r \rightarrow 0} f(r)=-\frac{1}{2}\left[\left(\lambda_{4}^{2}-\lambda_{3}^{2}\right) \lambda_{2}^{4}-\left(\lambda_{4}^{2}-\lambda_{2}^{2}\right) \lambda_{3}^{4}+\left(\lambda_{3}^{2}-\lambda_{2}^{2}\right) \lambda_{4}^{4}\right]
$$


By substituting Eq. (121) into (119) it is obtained

$$
\tilde{G}_{\alpha 3}^{*}=\tilde{U}_{\alpha}^{W}=\frac{r}{8 \pi} \frac{\left(-S_{w}+\rho_{w} k_{w} s\right) s}{(\lambda+2 \mu) k_{w}} \frac{x_{\alpha}}{r}(1-2 \ln (r))+O\left(r^{0}\right)
$$

With the same approach it is obtained

$$
\begin{gathered}
\tilde{G}_{\alpha 4}^{*}=\tilde{U}_{\alpha}^{A}=\frac{r}{8 \pi} \frac{\left(-S_{a}+\rho_{a} k_{a} s\right) s}{(\lambda+2 \mu) k_{a}} \frac{x_{\alpha}}{r}(1-2 \ln (r))+O\left(r^{0}\right) \\
\tilde{G}_{3 \beta}^{*}=\tilde{P}_{\beta}^{w S}=\frac{r}{8 \pi} \frac{F_{s}}{(\lambda+2 \mu) k_{w}} \frac{x_{\beta}}{r}(1-2 \ln (r))+O\left(r^{0}\right) \\
\tilde{G}_{4 \beta}^{*}=\tilde{P}_{\beta}^{a S}=\frac{r}{8 \pi} \frac{\left(1-F_{s}\right)}{(\lambda+2 \mu) k_{a}} \frac{x_{\beta}}{r}(1-2 \ln (r))+O\left(r^{0}\right) \\
\tilde{G}_{43}^{*}=\tilde{P}^{a W}=O\left(r^{0}\right) \\
\tilde{G}_{34}^{*}=\tilde{P}^{w A}=O\left(r^{0}\right)
\end{gathered}
$$

Finally we discuss $\tilde{G}_{\alpha \beta}^{*}=\tilde{U}_{\alpha \beta}^{s}$, which can be more compactly expressed as

$$
\begin{aligned}
\tilde{G}_{\alpha \beta}^{*}= & \tilde{U}_{\alpha \beta}^{s}=\frac{x_{\alpha} x_{\beta}}{2 \pi \mu r^{2}} \frac{-(\lambda+\mu) \Lambda^{2}}{\rho s^{2}} h(r)-\frac{\delta_{\alpha \beta}}{4 \pi \mu} \frac{-(\lambda+\mu) \Lambda^{2}}{\rho s^{2}} h(r)-\frac{\delta_{\alpha \beta}}{4 \pi \mu} \frac{-(\lambda+\mu) \Lambda^{2}}{\rho s^{2}} \\
& \left\{\begin{array}{l}
-\frac{\lambda_{1}^{2}\left(\lambda_{1}^{2}-K_{s s 1}^{2}\right)\left(\lambda_{1}^{2}-K_{s s 2}^{2}\right)}{\left(\lambda_{1}^{2}-\lambda_{2}^{2}\right)\left(\lambda_{1}^{2}-\lambda_{3}^{2}\right)\left(\lambda_{1}^{2}-\lambda_{4}^{2}\right)} K_{0}\left(\lambda_{1} r\right)-\frac{\lambda_{2}^{2}\left(\lambda_{2}^{2}-K_{s s 1}^{2}\right)\left(\lambda_{2}^{2}-K_{s s 2}^{2}\right)}{\left(\lambda_{2}^{2}-\lambda_{1}^{2}\right)\left(\lambda_{2}^{2}-\lambda_{3}^{2}\right)\left(\lambda_{2}^{2}-\lambda_{4}^{2}\right)} K_{0}\left(\lambda_{2} r\right) \\
-\frac{\lambda_{3}^{2}\left(\lambda_{3}^{2}-K_{s s 1}^{2}\right)\left(\lambda_{3}^{2}-K_{s s 2}^{2}\right)}{\left(\lambda_{3}^{2}-\lambda_{2}^{2}\right)\left(\lambda_{3}^{2}-\lambda_{1}^{2}\right)\left(\lambda_{3}^{2}-\lambda_{4}^{2}\right)} K_{0}\left(\lambda_{3} r\right)-\frac{\lambda_{4}^{2}\left(\lambda_{4}^{2}-K_{s s 1}^{2}\right)\left(\lambda_{4}^{2}-K_{s s 2}^{2}\right)}{\left(\lambda_{4}^{2}-\lambda_{2}^{2}\right)\left(\lambda_{4}^{2}-\lambda_{3}^{2}\right)\left(\lambda_{4}^{2}-\lambda_{1}^{2}\right)} K_{0}\left(\lambda_{4} r\right)
\end{array}\right\}+ \\
& +\frac{\delta_{i j}}{2 \pi \mu} K_{0}\left(\lambda_{1} r\right)
\end{aligned}
$$


Where

Table 1

Components

Singularity

\begin{tabular}{|c|c|}
\hline$\tilde{G}_{\alpha \beta}^{*}=\tilde{U}_{\alpha \beta}^{s}$ & weakly singular $(\ln (1 / r))$ \\
\hline$\tilde{G}_{\alpha 3}^{*}=\tilde{U}_{\alpha}^{W}$ & weakly singular $(\ln (1 / r))$ \\
\hline$\tilde{G}_{\alpha 4}^{*}=\tilde{U}_{\alpha}^{A}$ & weakly singular $(\ln (1 / r))$ \\
\hline$\tilde{G}_{3 \beta}^{*}=\tilde{P}_{\beta}^{w S}$ & weakly singular $(\ln (1 / r))$ \\
\hline$\tilde{G}_{4 \beta}^{*}=\tilde{P}_{\beta}^{a S}$ & weakly singular $(\ln (1 / r))$ \\
\hline$\tilde{G}_{33}^{*}=\tilde{P}^{w W}$ & weakly singular $(\ln (1 / r))$ \\
\hline$\tilde{G}_{44}^{*}=\tilde{P}^{a A}$ & weakly singular $(\ln (1 / r))$ \\
\hline$\tilde{G}_{43}^{*}=\tilde{P}^{a W}$ & regular (1) \\
\hline$\tilde{G}_{34}^{*}=\tilde{P}^{w A}$ & regular (1) \\
\hline$T_{\alpha \beta}^{s}$ & hyper singular $(1 / r)$ \\
\hline$T_{\alpha}^{W}$ & weakly singular $(\ln (1 / r))$ \\
\hline$T_{\alpha}^{A}$ & weakly singular $(\ln (1 / r))$ \\
\hline$\tilde{Q}_{\alpha}^{w S}$ & weakly singular $(\ln (1 / r))$ \\
\hline$\tilde{Q}_{\alpha}^{a s}$ & weakly singular $(\ln (1 / r))$ \\
\hline$\tilde{Q}^{w W}$ & hyper singular $(1 / r)$ \\
\hline$\tilde{Q}^{a A}$ & hyper singular $(1 / r)$ \\
\hline$\tilde{Q}^{w A}$ & regular (1) \\
\hline$\tilde{Q}^{a W}$ & regular (1) \\
\hline
\end{tabular}

(129)

$$
h(r)=\frac{1}{r^{2}}\left\{\begin{array}{l}
\frac{\lambda_{1}^{2}\left(\lambda_{1}^{2}-K_{s s 1}^{2}\right)\left(\lambda_{1}^{2}-K_{s s 2}^{2}\right)}{\left(\lambda_{1}^{2}-\lambda_{2}^{2}\right)\left(\lambda_{1}^{2}-\lambda_{3}^{2}\right)\left(\lambda_{1}^{2}-\lambda_{4}^{2}\right)} r^{2} K_{2}\left(\lambda_{1} r\right)+\frac{\lambda_{2}^{2}\left(\lambda_{2}^{2}-K_{s s 1}^{2}\right)\left(\lambda_{2}^{2}-K_{s s 2}^{2}\right)}{\left(\lambda_{1}^{2}-\lambda_{2}^{2}\right)\left(\lambda_{1}^{2}-\lambda_{3}^{2}\right)\left(\lambda_{1}^{2}-\lambda_{4}^{2}\right)} r^{2} K_{2}\left(\lambda_{2} r\right) \\
+\frac{\lambda_{3}^{2}\left(\lambda_{3}^{2}-K_{s s 1}^{2}\right)\left(\lambda_{3}^{2}-K_{s s 2}^{2}\right)}{\left(\lambda_{3}^{2}-\lambda_{2}^{2}\right)\left(\lambda_{3}^{2}-\lambda_{1}^{2}\right)\left(\lambda_{3}^{2}-\lambda_{4}^{2}\right)} r^{2} K_{2}\left(\lambda_{3} r\right)+\frac{\lambda_{4}^{2}\left(\lambda_{4}^{2}-K_{s s 1}^{2}\right)\left(\lambda_{4}^{2}-K_{s s 2}^{2}\right)}{\left(\lambda_{4}^{2}-\lambda_{2}^{2}\right)\left(\lambda_{4}^{2}-\lambda_{3}^{2}\right)\left(\lambda_{4}^{2}-\lambda_{1}^{2}\right)} r^{2} K_{2}\left(\lambda_{4} r\right)
\end{array}\right\}
$$


In order to determine the value of $h(r)$ when $r \rightarrow 0$, we take into account that $K_{2}(x)=\frac{2}{x^{2}}$ as $x \rightarrow 0$, this is the case 0 / 0 . We apply L'Hôpital's rule to Eq. (129), one obtains

$$
h(r)=-\frac{1}{2}
$$

For $r \rightarrow 0$ combining Eq. (130) and (128), and also keep in mind $K_{0}(x)=-\ln x$ as $x \rightarrow 0$, after some algebraic manipulation, we arrive at

$$
\tilde{G}_{\alpha \beta}^{*}=\tilde{U}_{\alpha \beta}^{s}=\underbrace{\frac{1}{8 \pi} \frac{1}{\mu(1-v)}\left\{-\frac{x_{\alpha} x_{\beta}}{r^{2}}-\delta_{\alpha \beta}(3-4 v) \ln (r)\right\}}_{\text {elastostatic fundamental solution }}+O\left(r^{0}\right)
$$

\section{Adjoint fundamental solution.}

$$
\begin{aligned}
& T_{\alpha \beta}^{S}= \frac{-1}{4 \pi} \frac{1}{(1-v) r}\left\{\frac{\partial r}{\partial n}\left[(1-2 v) \delta_{\alpha \beta}+2 r_{, \alpha} r_{, \beta}\right]-(1-2 v)\left(r_{, \alpha} n_{\beta}-r_{, \beta} n_{\alpha}\right)\right\}+ \\
& \frac{s r}{4 \pi(\lambda+2 \mu)} \frac{x_{\beta}}{r}\left[\frac{S_{w} F_{s}}{k_{w}}\left(\frac{1}{2}-\ln (r)\right)+\frac{S_{a}\left(1-F_{s}\right)}{k_{a}}\left(\frac{1}{2}-\ln (r)\right)\right) n_{\alpha}+O\left(r^{0}\right) \\
& T_{\alpha}^{W}= \frac{\mu}{4 \pi} \frac{\left(-S_{w}\right) s}{(\lambda+2 \mu) k_{w}}\left[(1+2 \ln (r)) n_{\alpha}-2 r_{, \alpha} r_{, n}\right]+ \\
& \frac{\mu}{4 \pi} \frac{\left(\rho_{w} k_{w}\right) s^{2}}{(\lambda+2 \mu) k_{w}}\left[\left(1-2\left(\frac{\mu+\lambda}{\mu}\right) \ln (r)\right) n_{\alpha}-2 r_{, \alpha} r_{, n}\right]+O\left(r^{0}\right) \\
& T_{\alpha}^{A}= \frac{\mu}{4 \pi} \frac{\left(-S_{a}\right) s}{(\lambda+2 \mu) k_{a}}\left[(1+2 \ln (r)) n_{\alpha}-2 r_{, \alpha} r_{, n}\right]+ \\
& \frac{\mu}{4 \pi} \frac{\left(\rho_{a} k_{a} s\right) s}{(\lambda+2 \mu) k_{a}}\left[\left(1-2\left(\frac{\mu+\lambda}{\mu}\right) \ln (r)\right) n_{\alpha}-2 r_{, \alpha} r_{, n}\right]+O\left(r^{0}\right)
\end{aligned}
$$




$$
\begin{gathered}
\tilde{Q}_{\alpha}^{w S}=\frac{1}{8 \pi} \frac{F_{s}}{(\lambda+2 \mu)}\left[(1-2 \ln (r)) n_{\alpha}-2 r_{, \alpha} r_{, n}\right]+O\left(r^{0}\right) \\
\tilde{Q}_{\alpha}^{a S}=\frac{1}{8 \pi} \frac{\left(1-F_{s}\right)}{(\lambda+2 \mu)}\left[(1-2 \ln (r)) n_{\alpha}-2 r_{, \alpha} r_{, n}\right]+O\left(r^{0}\right) \\
\tilde{Q}^{w W}=\underbrace{\frac{-r_{, n}}{2 \pi r}}_{\text {acoustic fundamental solution }}+O\left(r^{0}\right) \\
\tilde{Q}^{a A}=\quad \underbrace{\frac{-r_{, n}}{2 \pi r}}_{\text {acoustic fundamental solution }}+O\left(r^{0}\right) \\
\tilde{Q}^{w A}=O\left(r^{0}\right) \\
\tilde{Q}^{a W}=O\left(r^{0}\right)
\end{gathered}
$$




\section{Analytical verification of the fundamental solutions}

Having derived the fundamental solution, at this stage, it is of interest to verify the validity of these solutions in some what more detail. 1 limiting case is presented here. Investigate the solution form as $k_{w}$ and $k_{a}$ approach infinity, $\rho_{w}, \rho_{a}$ and $F_{s}$ approach zero, to see if they would exactly take the same form as elastodynamic fundamental solution in Laplace transform domain.

\subsection{Limiting Case: Elastodynamic}

Letting $k_{w}$ and $k_{a}$ approach infinity and $\rho_{w}, \rho_{a}$ and $F_{s}$ equal zero, the roots of the determinant equation (85) reduce to two and we will have

$$
\begin{gathered}
\lambda_{1}^{2}=\frac{\rho}{\mu} \cdot s^{2}, \lambda_{3}^{2}=\lambda_{2}^{2}=0 \text { and } \lambda_{4}^{2}=\Lambda^{2}=\frac{\rho}{(\lambda+2 \mu)} \cdot s^{2} \\
K_{s s 1}^{2}=K_{s s 2}^{2}=0
\end{gathered}
$$

Then

$$
\begin{gathered}
\tilde{G}_{3 j}=\tilde{G}_{4 j}=0 \\
\tilde{G}_{\alpha 3}=\tilde{G}_{\alpha 4}=0 \\
\tilde{G}_{i j}^{*}=\tilde{G}_{i j}=\frac{1}{2 \pi \rho C_{2}^{2}}\left(a \delta_{i j}-b \frac{x_{i} x_{j}}{r^{2}}\right)
\end{gathered}
$$

Where

$$
\begin{gathered}
a=K_{0}\left(\frac{s \cdot r}{C_{2}}\right)+\frac{C_{2}}{s \cdot r}\left(K_{1}\left(\frac{s \cdot r}{C_{2}}\right)-\frac{C_{2}}{C_{1}} K_{1}\left(\frac{s \cdot r}{C_{1}}\right)\right) \\
b=K_{2}\left(\frac{s \cdot r}{C_{2}}\right)-\frac{C_{2}^{2}}{C_{1}^{2}} K_{2}\left(\frac{s \cdot r}{C_{1}}\right)
\end{gathered}
$$




$$
\begin{gathered}
C_{1}^{2}=\frac{(\lambda+2 \mu)}{\rho} \\
C_{2}^{2}=\frac{\mu}{\rho}
\end{gathered}
$$

Equations (143) to (145) show the fundamental singular solutions in the Laplace transform domain for a point force in 2-D solid of infinite extent. This limiting case supports that the Laplace transform domain fundamental solutions of dynamic unsaturated poroelasticity for 2$\mathrm{D}$ cases derived in previous sections are likely to be correct.

\section{Visualization of some fundamental solution}

Here, some fundamental solutions are calculated to bring to mind their general behaviour.

An unsaturated soil with incompressible solid grains is considered in which the material properties were defined in the metric system as follows:

Table 2. Material data of an unsaturated soil (Mechanical parameters)

\begin{tabular}{ccccccccc}
\hline $\mathrm{K}_{\mathrm{b}}(-)$ & $\mathrm{E}\left(\mathrm{N} / \mathrm{m}^{2}\right)$ & $\mathrm{K}_{\mathrm{l}}(-)$ & $\mathrm{a}_{\mathrm{e}}(-)$ & $\mathrm{b}_{\mathrm{e}}(-)$ & $\mathrm{e}_{0}(-)$ & $\sigma_{\mathrm{e}}(-)$ & $\mathrm{n}(-)$ & $\rho_{\mathrm{s}}\left(\mathrm{Kg} / \mathrm{m}^{3}\right)$ \\
\hline 3281.0 & $21 \times 10^{6}$ & 1678 & 2.0 & 0.2 & 0.4 & $35 \times 10^{5}$ & 0.4 & 2600
\end{tabular}

Table 3. Material data of an unsaturated soil (Water parameters)

\begin{tabular}{cccccccc}
\hline$\rho_{\mathrm{w}}\left(\mathrm{Kg} / \mathrm{m}^{3}\right)$ & $\mathrm{K}_{\mathrm{w}}\left(\mathrm{N} / \mathrm{m}^{2}\right)$ & $\mathrm{a}_{\mathrm{w}}(\mathrm{m} / \mathrm{s})$ & $\alpha_{\mathrm{w}}(-)$ & $\mathrm{S}_{\mathrm{wu}}(-)$ & $\beta_{\mathrm{w}}\left(\mathrm{Pa}^{-1}\right)$ & $\mathrm{m}_{\mathrm{suc}}(-)$ & $\mathrm{P}_{\mathrm{w}}(\mathrm{Pa})$ \\
\hline 1000.0 & $2.15 \times 10^{9}$ & $1.2 \times 10^{-9}$ & 5.0 & 0.05 & $1.08 \times 10^{-8}$ & 1 & $300 \times 10^{5}$ \\
\hline
\end{tabular}

Table 4. Material data of an unsaturated soil (Air parameters)

\begin{tabular}{ccccccc}
\hline$\rho_{\mathrm{a}}\left(\mathrm{Kg} / \mathrm{m}^{3}\right)$ & $\mathrm{P}_{\mathrm{atm}}\left(\mathrm{N} / \mathrm{m}^{2}\right)$ & $\mathrm{K}_{\mathrm{a}}\left(\mathrm{N} / \mathrm{m}^{2}\right)$ & $\mathrm{b}_{\mathrm{a}}\left(\mathrm{m}^{2}\right)$ & $\alpha_{\mathrm{a}}(-)$ & $\mu_{\mathrm{a}}\left(\mathrm{N} . \mathrm{s}^{2} \mathrm{~m}^{-2}\right)$ & $\mathrm{P}_{\mathrm{a}}(\mathrm{Pa})$ \\
\hline 1.0 & $10^{5}$ & $1.01 \times 10^{5}$ & $3 \times 10^{-10}$ & 4.0 & $1.846 \times 10^{-5}$ & 0.0 \\
\hline
\end{tabular}


Firstly in Fig. 3, the displacement in direction 1 due to a unit point force in the same direction $\tilde{U}_{11}^{s}$ is depicted in 3D versus the distance $r$ and the frequency $\omega$. For this aim, the real part of the complex Laplace variable $s$ is set to zero, i.e., $s=i \omega$.

The absolute value of the complex valued displacement solutions, i.e., the amplitude, is presented. Because of the singularity of the solutions, the figure is clipped in the range of $10^{-10}$. As shown in this figure, the singular behaviour for small values of $r$ is roughly independent of the frequency. Also, for a constant $r$ away from the origin, a wave like form decrease of amplitudes is observed by increasing the frequencies. These observations are in agreement with saturated displacement fundamental solution [48].

Abs $\amalg^{4}$

Figure 3. Displacement fundamental solution $\operatorname{abs}\left(\tilde{U}_{11}^{s}\right)$ in direction 1 due to a unit point force in the same direction versus frequency, $\omega$ and distance, $r$.

Also, the 2D visualizations of the some fundamental solutions are presented by keeping constant the distance $r$ and varying the frequency $\omega$ to have a better insight into the behaviour of the fundamental solutions. In addition, all results, i.e., the displacement and pressure results are normalized to their singular behaviour presented in section 4 . 


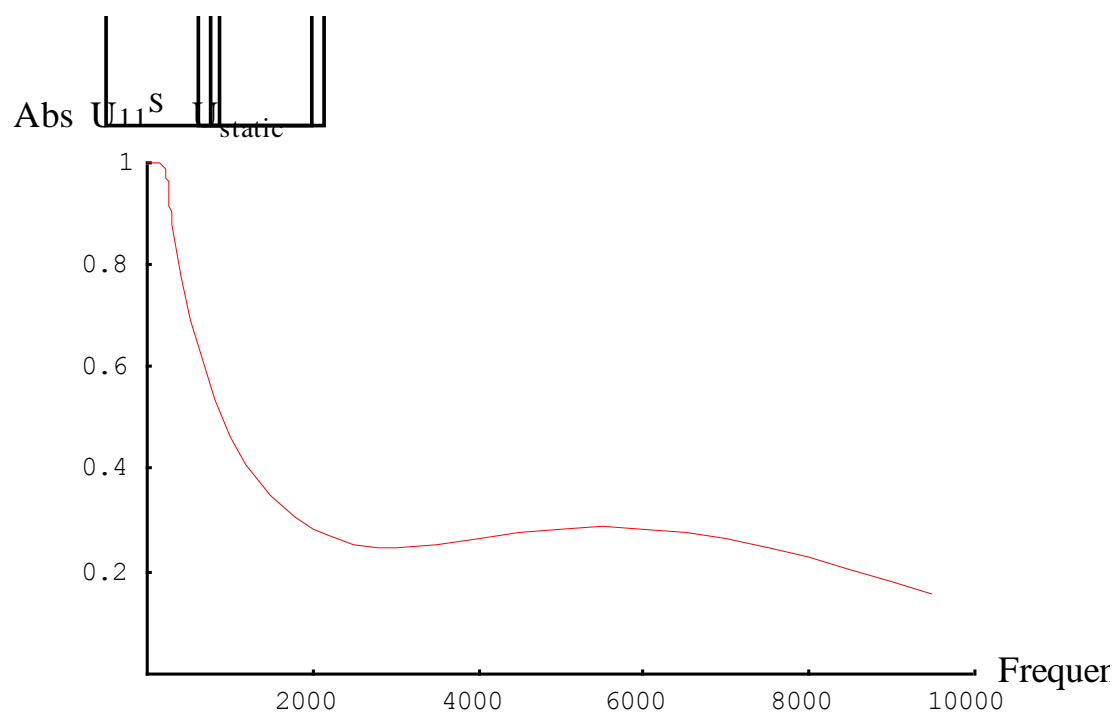

(a) $\mathrm{r}=0.1 \mathrm{~m}$

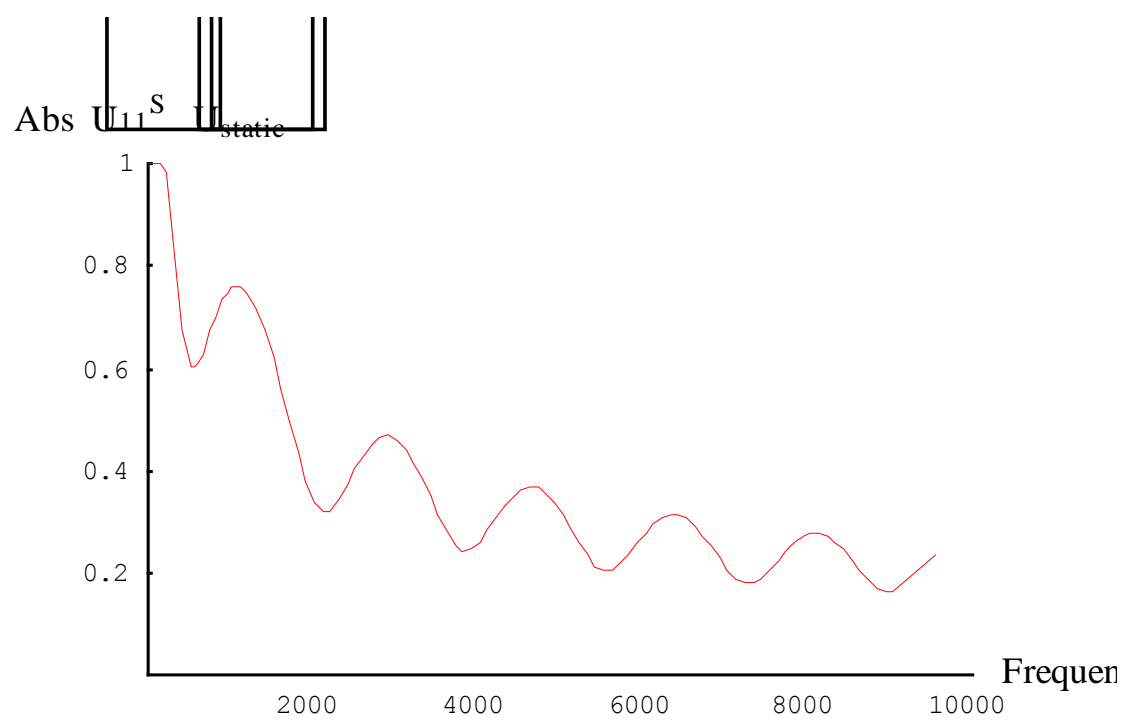

(b) $\mathrm{r}=0.5 \mathrm{~m}$

Figure 4. Displacement fundamental solution $\operatorname{abs}\left(\tilde{U}_{11}^{s}\right)$ normalized with $\tilde{U}_{\text {static }}$ versus frequency $\omega$.

In Figure 4, the normalized displacement fundamental solution $\operatorname{abs}\left(\tilde{U}_{11}^{s}\right)$ is presented versus frequency for two points at $r=0.1 \mathrm{~m}$ and at $r=0.5 \mathrm{~m}$ distance from the origin.

A comparison between Figure (2a) and Figure (2b) shows that there is a difference between the arrival times of the fast compressional wave. It is evident that the longer distance away, 
the later arrival time, i.e., shorter frequency. Then in Figure (2b) at $r=0.5 \mathrm{~m}$, the fast compressional wave arrives later and influences the shorter frequencies.

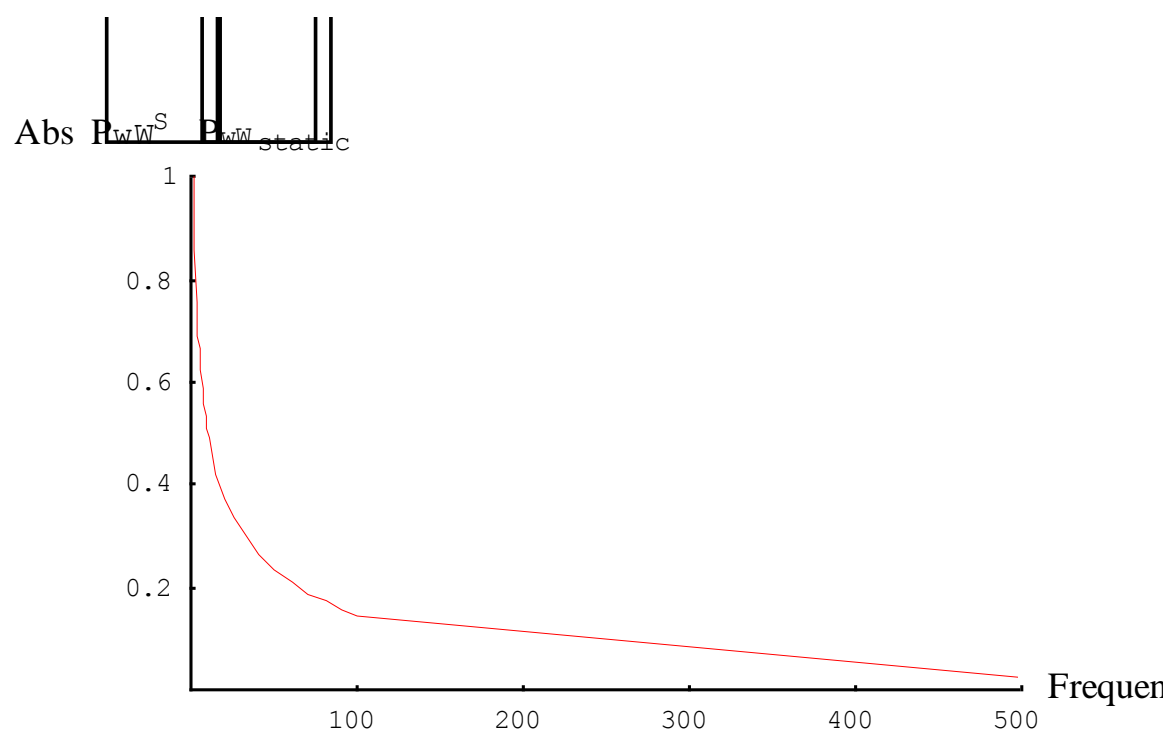

(a) $\mathrm{r}=0.1 \mathrm{~m}$

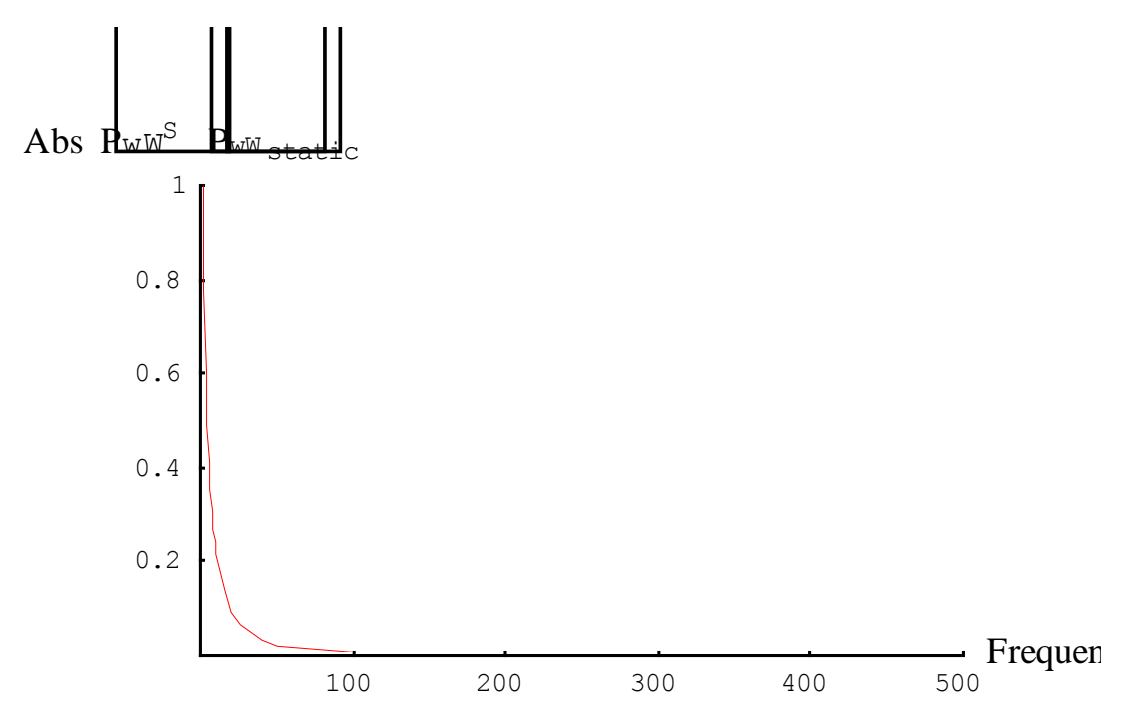

(b) $\mathrm{r}=0.5 \mathrm{~m}$

Figure 5. Water pressure fundamental solution $\operatorname{abs}\left(\tilde{P}^{w W}\right)$ normalized with $\tilde{P}_{\text {staic }}^{w W}$

Next, in Figure 5, the normalized water pressure due to a source in the water fluid is depicted. As shown, a change in pressure is immediately in two cases. Therefore, the pressure can not show a strong time, respectively frequency, dependence [48]. 


\section{Conclusion}

In this paper, firstly coupled governing differential equations of a porous medium saturated by two compressible fluids (water and air) subjected to dynamic loadings is presented based on the poromechanics theory in the frame of the suction-based mathematical model presented by Gatmiri [14] and Gatmiri et al. [15]. After that, the Boundary Integral Equation (BIE) is developed directly from those equations via the use of weighted residuals method for the first time. Finally, the associated fundamental solution in Laplace transformed domain is presented by the use of the method of Hörmander or Kupradze for $2 \mathrm{D} u_{i}-p_{w}-p_{a}$ formulation of unsaturated porous media. Also, the singular behaviour of the fundamental solutions is studied in order to be able to determine the unknown boundary data. It is observed that the singularity of these solutions is equal to the elastostatic, poroelastostayic or the acoustic fundamental solutions.

The derived Laplace transform domain fundamental solutions could be directly implemented in time domain BEM in which the convolution integral is numerically approximated by a new approach so-called “Operational Quadrature Methods" developed by Lubich [35, 36] for modelling the transient behaviour of unsaturated porous media and enables one to develop more effective numerical hybrid $\mathrm{BE} / \mathrm{FE}$ methods for solving $2 \mathrm{D}$ nonlinear wave propagation problems in the near future. 


\section{Appendix A}

Coefficients of determinant of $\tilde{\mathbf{B}}^{*}$

$$
\begin{aligned}
& D_{1}=\mu(\lambda+2 \mu) k_{w} k_{a} \\
& D_{2} / D_{1}=-\frac{\rho}{\mu}-\frac{\rho}{(\lambda+2 \mu)}-\frac{F_{s} \rho_{w}}{(\lambda+2 \mu)}-\frac{\rho_{a}\left(1-F_{s}\right)}{(\lambda+2 \mu)} \\
& D_{3} / D_{1}=\frac{C_{a a}}{k_{a}}+\frac{C_{w w}}{k_{w}}+\frac{S_{w} F_{s}}{(\lambda+2 \mu) k_{w}}+\frac{S_{a}\left(1-F_{s}\right)}{(\lambda+2 \mu) k_{a}} \\
& D_{4} / D_{1}=\frac{\rho^{2}}{\mu(\lambda+2 \mu)}+\frac{\rho \rho_{w} F_{s}}{\mu(\lambda+2 \mu)}+\frac{\rho \rho_{a}\left(1-F_{s}\right)}{\mu(\lambda+2 \mu)} \\
& D_{5} / D_{1}=-\frac{\rho C_{a a}}{\mu k_{a}}-\frac{\rho C_{w w}}{\mu k_{w}}-\frac{\rho S_{w} F_{s}}{\mu(\lambda+2 \mu) k_{w}}-\frac{\rho S_{a}\left(1-F_{s}\right)}{\mu(\lambda+2 \mu) k_{a}}-\frac{\rho C_{a a}}{(\lambda+2 \mu) k_{a}} \\
& -\frac{\rho C_{w w}}{(\lambda+2 \mu) k_{w}}-\frac{\rho_{w}\left(F_{s} C_{a a}-\left(1-F_{s}\right) C_{w a}\right)}{(\lambda+2 \mu) k_{a}}-\frac{\rho_{a}\left(-F_{s} C_{w a}+\left(1-F_{s}\right) C_{w w}\right)}{(\lambda+2 \mu) k_{w}} \\
& D_{6} / D_{1}=\frac{\left(C_{w w} C_{a a}-C_{w a}^{2}\right)}{k_{w} k_{a}}+\frac{S_{w}\left(F_{s} C_{a a}-C_{w a}\left(1-F_{s}\right)\right)}{(\lambda+2 \mu) k_{w} k_{a}}+\frac{S_{a}\left(-F_{s} C_{w a}+C_{w w}\left(1-F_{s}\right)\right)}{(\lambda+2 \mu) k_{w} k_{a}} \\
& D_{7} / D_{1}=\frac{\rho^{2} C_{a a}}{\mu(\lambda+2 \mu) k_{a}}+\frac{\rho^{2} C_{w w}}{\mu(\lambda+2 \mu) k_{w}}+\frac{\rho \rho_{w} F_{s} C_{a a}}{\mu(\lambda+2 \mu) k_{a}}-\frac{\rho \rho_{w} C_{w a}\left(1-F_{s}\right)}{\mu(\lambda+2 \mu) k_{a}} \\
& +\frac{\rho \rho_{a}\left(-F_{s} C_{w a}+C_{w w}\left(1-F_{s}\right)\right)}{\mu(\lambda+2 \mu) k_{w}} \\
& D_{8} / D_{1}=\frac{-\rho\left(C_{w w} C_{a a}-C_{w a}^{2}\right)}{\mu k_{w} k_{a}}-\frac{\rho\left(C_{w w} C_{a a}-C_{w a}^{2}\right)}{(\lambda+2 \mu) k_{w} k_{a}}-\frac{\rho S_{w}\left(F_{s} C_{a a}-C_{w a}\left(1-F_{s}\right)\right)}{\mu(\lambda+2 \mu) k_{w} k_{a}} \\
& -\rho S_{a} \frac{\left(-F_{s} C_{w a}+C_{w w}\left(1-F_{s}\right)\right)}{\mu(\lambda+2 \mu) k_{w} k_{a}} \\
& D_{9} / D_{1}=\frac{\rho^{2}\left(C_{w w} C_{a a}-C_{w a}^{2}\right)}{\mu(\lambda+2 \mu) k_{w} k_{a}}=\frac{\rho}{\mu} \frac{\rho}{(\lambda+2 \mu)}\left[\frac{C_{w w} C_{a a}}{k_{w} k_{a}}-\frac{C_{w a}^{2}}{k_{w} k_{a}}\right]
\end{aligned}
$$




\section{Appendix B}

The components of cofactors matrix $\tilde{\mathbf{B}}^{* \text { co }}$ are obtained as following

$$
\begin{gathered}
\tilde{B}_{\alpha \beta}^{* \text { co }}=\left[C_{8} \nabla^{4}+\left(C_{9}+C_{10} s\right) s \nabla^{2}+\left(C_{11}+C_{12} s\right) s^{2}\right] \partial_{1} \partial_{2}+ \\
\delta_{i j}(\lambda+2 \mu) k_{w} k_{a}\left(\Delta-\lambda_{2}^{2}\right)\left(\Delta-\lambda_{3}^{2}\right)\left(\Delta-\lambda_{4}^{2}\right) \\
\tilde{B}_{\alpha 3}^{* c 0}=\left(\left(C_{13}+C_{14} s\right) s \nabla^{4}+\left(C_{15}+C_{16} s+C_{17} s^{2}\right) s^{2} \nabla^{2}+\left(C_{18}+C_{19} s\right) s^{4}\right) \partial_{i} \\
\tilde{B}_{\alpha 4}^{* c o}=\left(\left(C_{20}+C_{21} s\right) s \nabla^{4}+\left(C_{22}+C_{23} s+C_{24} s^{2}\right) s^{2} \nabla^{2}+\left(C_{25}+C_{26} s\right) s^{4}\right) \partial_{i} \\
\tilde{B}_{3 \beta}^{* c o}=\left(C_{27} \nabla^{4}+\left(C_{28}+C_{29} s\right) s \nabla^{2}+C_{30} s^{3}\right) \partial_{i} \\
\tilde{B}_{4 \beta}^{* c o}=\left(C_{42} \nabla^{4}+\left(C_{43}+C_{44} s\right) s \nabla^{2}+C_{45} s^{3}\right) \partial_{i} \\
\tilde{B}_{34}^{* c o}=+\left(C_{37}+C_{38} s\right) s \nabla^{4}+\left(C_{39}+C_{40} s\right) s^{3} \nabla^{2}+C_{41} s^{5} \\
\tilde{B}_{43}^{* c o}=\left(C_{46}+C_{47} s\right) s \nabla^{4}+\left(C_{48}+C_{49} s\right) s^{3} \nabla^{2}+C_{50} s^{5} \\
\tilde{B}_{33}^{* c o}=C_{31} \nabla^{6}+\left(C_{32}+C_{33} s\right) s \nabla^{4}+\left(C_{34}+C_{35} s\right) s^{3} \nabla^{2}+C_{36} s^{5} \\
\tilde{B}_{44}^{* c o}=C_{51} \nabla^{6}+\left(C_{52}+C_{53} s\right) s \nabla^{4}+\left(C_{54}+C_{55} s\right) s^{3} \nabla^{2}+C_{56} s^{5}
\end{gathered}
$$

where $C_{8}=-a_{2} a_{9} a_{13}, C_{9}=a_{4} a_{8} a_{13}+a_{6} a_{9} a_{12}-a_{2}\left(a_{9} a_{14}+a_{10} a_{13}\right), \quad C_{10}=a_{5} a_{8} a_{13}+a_{7} a_{9} a_{12}$, $C_{11}=-a_{2}\left(-a_{11}^{2}+a_{10} a_{14}\right)-a_{4}\left(a_{11} a_{12}-a_{8} a_{14}\right)-a_{6}\left(a_{8} a_{11}-a_{10} a_{12}\right)$

$C_{12}=-a_{5}\left(a_{11} a_{12}-a_{8} a_{14}\right)-a_{7}\left(a_{8} a_{11}-a_{10} a_{12}\right) \quad, \quad C_{13}=-a_{1} a_{4} a_{13} \quad, \quad C_{14}=-a_{1} a_{5} a_{13}$, $C_{15}=a_{1}\left(a_{6} a_{11}-a_{4} a_{14}\right) \quad, \quad C_{16}=a_{1}\left(a_{7} a_{11}-a_{5} a_{14}\right)-a_{3} a_{4} a_{13} \quad, \quad C_{17}=-a_{3} a_{5} a_{13} \quad$, $C_{18}=a_{3}\left(a_{6} a_{11}-a_{4} a_{14}\right) \quad, \quad C_{19}=a_{3}\left(a_{7} a_{11}-a_{5} a_{14}\right) \quad, \quad C_{20}=-a_{1} a_{6} a_{9} \quad, \quad C_{21}=-a_{1} a_{7} a_{9}$, $C_{22}=a_{1}\left(a_{4} a_{11}-a_{6} a_{10}\right), C_{23}=a_{1}\left(a_{5} a_{11}-a_{7} a_{10}\right)-a_{3} a_{6} a_{9}, C_{24}=-a_{3} a_{7} a_{9}, C_{25}=a_{3}\left(a_{4} a_{11}-a_{6} a_{10}\right)$, $C_{26}=a_{3}\left(a_{5} a_{11}-a_{7} a_{10}\right) \quad, \quad C_{27}=-a_{1} a_{8} a_{13} \quad, \quad C_{28}=a_{1}\left(a_{11} a_{12}-a_{8} a_{14}\right) \quad, \quad C_{29}=-a_{3} a_{8} a_{13} \quad$, $C_{30}=a_{3}\left(a_{11} a_{12}-a_{8} a_{14}\right) \quad, \quad C_{31}=a_{1}\left(a_{1}+a_{2}\right) a_{13} \quad, \quad C_{32}=-a_{1} a_{6} a_{12}+a_{1}\left(a_{1}+a_{2}\right) a_{14}$, $C_{33}=\left(a_{1}+a_{2}\right) a_{3} a_{13}+a_{1}\left(a_{3} a_{13}-a_{7} a_{12}\right) \quad, \quad C_{34}=-a_{3} a_{6} a_{12}+\left(a_{1}+a_{2}\right) a_{3} a_{14}+a_{1} a_{3} a_{14}$, $C_{35}=-a_{3} a_{7} a_{12}+a_{3}^{2} a_{13} \quad, \quad C_{36}=a_{3}^{2} a_{14} \quad, \quad C_{37}=a_{1} a_{6} a_{8}-a_{1}\left(a_{1}+a_{2}\right) a_{11} \quad, \quad C_{38}=a_{1} a_{7} a_{8} \quad$, $C_{39}=a_{3} a_{6} a_{8}-\left(a_{1}+a_{2}\right) a_{3} a_{11}-a_{1} a_{3} a_{11} \quad, \quad C_{40}=a_{3} a_{7} a_{8} \quad, \quad C_{41}=-a_{3}^{2} a_{11} \quad, \quad C_{42}=-a_{1} a_{9} a_{12} \quad$, 


$$
\begin{aligned}
& C_{43}=a_{1}\left(a_{8} a_{11}-a_{10} a_{12}\right), \quad C_{44}=-a_{3} a_{9} a_{12}, \quad C_{45}=+a_{3}\left(a_{8} a_{11}-a_{10} a_{12}\right), \\
& C_{46}=a_{1} a_{4} a_{12}-a_{1}\left(a_{1}+a_{2}\right) a_{11}, C_{47}=a_{1} a_{5} a_{12}, C_{48}=a_{3} a_{4} a_{12}-\left(a_{1}+a_{2}\right) a_{3} a_{11}-a_{1} a_{3} a_{11}, \\
& C_{49}=a_{3} a_{5} a_{12}, \quad C_{50}=-a_{3}^{2} a_{11}, C_{51}=a_{1}\left(a_{1}+a_{2}\right) a_{9}, C_{52}=-a_{1} a_{4} a_{8}+a_{1}\left(a_{1}+a_{2}\right) a_{10}, \\
& C_{53}=\left(a_{1}+a_{2}\right) a_{3} a_{9}+a_{1} a_{3} a_{9}-a_{1} a_{5} a_{8}, \\
& C_{55}=a_{3}^{2} a_{9}-a_{3} a_{5} a_{8} \text { and } C_{56}=a_{3}^{2} a_{10}, C_{54}=-a_{3} a_{4} a_{8}+\left(a_{1}+a_{2}\right) a_{3} a_{10}+a_{1} a_{3} a_{10},
\end{aligned}
$$




\section{Appendix C}

$$
\begin{aligned}
& C_{1}^{s S}=\frac{1}{2 \pi \mu} \frac{-(\lambda+\mu) \Lambda^{2}}{\rho s^{2}} \frac{\left(\lambda_{1}^{2}-K_{s s 1}^{2}\right)\left(\lambda_{1}^{2}-K_{s s 2}^{2}\right)}{\left(\lambda_{1}^{2}-\lambda_{2}^{2}\right)\left(\lambda_{1}^{2}-\lambda_{3}^{2}\right)\left(\lambda_{1}^{2}-\lambda_{4}^{2}\right)} \\
& C_{2}^{s S}=\frac{1}{2 \pi \mu} \frac{-(\lambda+\mu) \Lambda^{2}}{\rho s^{2}} \frac{\left(\lambda_{2}^{2}-K_{s s 1}^{2}\right)\left(\lambda_{2}^{2}-K_{s s 2}^{2}\right)}{\left(\lambda_{2}^{2}-\lambda_{1}^{2}\right)\left(\lambda_{2}^{2}-\lambda_{4}^{2}\right)\left(\lambda_{2}^{2}-\lambda_{3}^{2}\right)} \\
& C_{3}^{s S}=\frac{1}{2 \pi \mu} \frac{-(\lambda+\mu) \Lambda^{2}}{\rho s^{2}} \frac{\left(\lambda_{3}^{2}-K_{s s 1}^{2}\right)\left(\lambda_{3}^{2}-K_{s s 2}^{2}\right)}{\left(\lambda_{3}^{2}-\lambda_{4}^{2}\right)\left(\lambda_{3}^{2}-\lambda_{2}^{2}\right)\left(\lambda_{3}^{2}-\lambda_{1}^{2}\right)} \\
& C_{4}^{s S}=\frac{1}{2 \pi \mu} \frac{-(\lambda+\mu) \Lambda^{2}}{\rho s^{2}} \frac{\left(\lambda_{4}^{2}-K_{s s 1}^{2}\right)\left(\lambda_{4}^{2}-K_{s s 2}^{2}\right)}{\left(\lambda_{4}^{2}-\lambda_{3}^{2}\right)\left(\lambda_{4}^{2}-\lambda_{1}^{2}\right)\left(\lambda_{4}^{2}-\lambda_{2}^{2}\right)} \\
& C_{5}^{s S}=\frac{\delta_{\alpha \beta}}{2 \pi \mu} \\
& C_{1}^{s W}= \\
& \frac{\left(S_{w}-\rho_{w} k_{w} s\right) s}{2 \pi(\lambda+2 \mu) k_{w}\left(\lambda_{4}^{2}-\lambda_{2}^{2}\right)\left(\lambda_{3}^{2}-\lambda_{2}^{2}\right)}\left(\lambda_{2}^{2}-\frac{\left(C_{w a}\left(S_{a}-\rho_{a} k_{a} s\right) s-C_{a a}\left(S_{w}-\rho_{w} k_{w} s\right) s\right)}{k_{a}\left(S_{w}-\rho_{w} k_{w} s\right)}\right) \\
& C_{2}^{s W}= \\
& \frac{\left(S_{w}-\rho_{w} k_{w} s\right) s}{2 \pi(\lambda+2 \mu) k_{w}\left(\lambda_{4}^{2}-\lambda_{3}^{2}\right)\left(\lambda_{2}^{2}-\lambda_{3}^{2}\right)}\left(\lambda_{3}^{2}-\frac{\left(C_{w a}\left(S_{a}-\rho_{a} k_{a} s\right) s-C_{a a}\left(S_{w}-\rho_{w} k_{w} s\right) s\right)}{k_{a}\left(S_{w}-\rho_{w} k_{w} s\right)}\right) \\
& C_{3}^{s W}= \\
& \frac{\left(S_{w}-\rho_{w} k_{w} s\right) s}{2 \pi(\lambda+2 \mu) k_{w}\left(\lambda_{3}^{2}-\lambda_{4}^{2}\right)\left(\lambda_{2}^{2}-\lambda_{4}^{2}\right)}\left(\lambda_{4}^{2}-\frac{\left(C_{w a}\left(S_{a}-\rho_{a} k_{a} s\right) s-C_{a a}\left(S_{w}-\rho_{w} k_{w} s\right) s\right)}{k_{a}\left(S_{w}-\rho_{w} k_{w} s\right)}\right) \\
& C_{1}^{s A}= \\
& \frac{\left(S_{a}-\rho_{a} k_{a} s\right) s}{2 \pi(\lambda+2 \mu) k_{a}\left(\lambda_{4}^{2}-\lambda_{2}^{2}\right)\left(\lambda_{3}^{2}-\lambda_{2}^{2}\right)}\left(\lambda_{2}^{2}-\frac{\left(C_{w a}\left(S_{w}-\rho_{w} k_{w} s\right) s-C_{w w}\left(S_{a}-\rho_{a} k_{a} s\right) s\right)}{k_{w}\left(S_{a}-\rho_{a} k_{a} s\right)}\right) \text { (C.10) } \\
& C_{2}^{s A}= \\
& \frac{\left(S_{a}-\rho_{a} k_{a} s\right) s}{2 \pi(\lambda+2 \mu) k_{a}\left(\lambda_{4}^{2}-\lambda_{3}^{2}\right)\left(\lambda_{2}^{2}-\lambda_{3}^{2}\right)}\left(\lambda_{3}^{2}-\frac{\left(C_{w a}\left(S_{w}-\rho_{w} k_{w} s\right) s-C_{w w}\left(S_{a}-\rho_{a} k_{a} s\right) s\right)}{k_{w}\left(S_{a}-\rho_{a} k_{a} s\right)}\right) \\
& C_{3}^{s A}= \\
& \frac{\left(S_{a}-\rho_{a} k_{a} s\right) s}{2 \pi(\lambda+2 \mu) k_{a}\left(\lambda_{3}^{2}-\lambda_{4}^{2}\right)\left(\lambda_{2}^{2}-\lambda_{4}^{2}\right)}\left(\lambda_{4}^{2}-\frac{\left(C_{w a}\left(S_{w}-\rho_{w} k_{w} s\right) s-C_{w w}\left(S_{a}-\rho_{a} k_{a} s\right) s\right)}{k_{w}\left(S_{a}-\rho_{a} k_{a} s\right)}\right)
\end{aligned}
$$




$$
\begin{aligned}
& C_{1}^{w S}=-\frac{F_{s}}{2 \pi(\lambda+2 \mu)\left(\lambda_{4}^{2}-\lambda_{2}^{2}\right)\left(\lambda_{3}^{2}-\lambda_{2}^{2}\right)}\left(\lambda_{2}^{2}-\frac{\left(C_{w a}\left(1-F_{s}\right)-F_{s} C_{a a}\right)}{F_{s} k_{a}} s\right) \\
& C_{2}^{w S}=-\frac{F_{s}}{2 \pi(\lambda+2 \mu)\left(\lambda_{4}^{2}-\lambda_{3}^{2}\right)\left(\lambda_{2}^{2}-\lambda_{3}^{2}\right)}\left(\lambda_{3}^{2}-\frac{\left(C_{w a}\left(1-F_{s}\right)-F_{s} C_{a a}\right)}{F_{s} k_{a}} s\right) \\
& C_{3}^{w S}=-\frac{F_{s}}{2 \pi(\lambda+2 \mu)\left(\lambda_{3}^{2}-\lambda_{4}^{2}\right)\left(\lambda_{2}^{2}-\lambda_{4}^{2}\right)}\left(\lambda_{4}^{2}-\frac{\left(C_{w a}\left(1-F_{s}\right)-F_{s} C_{a a}\right)}{F_{s} k_{a}} s\right) \\
& C_{1}^{w W}=\frac{1}{2 \pi} \frac{1}{\left(\lambda_{4}^{2}-\lambda_{2}^{2}\right)\left(\lambda_{3}^{2}-\lambda_{2}^{2}\right)}\left(\lambda_{2}^{2}-K_{w}^{2}\right)\left(\lambda_{2}^{2}-\Lambda^{2}\right) \\
& C_{2}^{w W}=\frac{1}{2 \pi} \frac{1}{\left(\lambda_{4}^{2}-\lambda_{3}^{2}\right)\left(\lambda_{2}^{2}-\lambda_{3}^{2}\right)}\left(\lambda_{3}^{2}-K_{w}^{2}\right)\left(\lambda_{3}^{2}-\Lambda^{2}\right) \\
& C_{3}^{w W}=\frac{1}{2 \pi} \frac{1}{\left(\lambda_{3}^{2}-\lambda_{4}^{2}\right)\left(\lambda_{2}^{2}-\lambda_{4}^{2}\right)}\left(\lambda_{4}^{2}-K_{w}^{2}\right)\left(\lambda_{4}^{2}-\Lambda^{2}\right) \\
& C_{1}^{w A}=\frac{s}{2 \pi(\lambda+2 \mu) k_{a}} \frac{\left(-(\lambda+2 \mu) C_{w a}+\left(\rho_{a} k_{a} s-S_{a}\right) F_{s}\right) \lambda_{2}^{2}+\rho C_{w a} s^{2}}{\left(\lambda_{4}^{2}-\lambda_{2}^{2}\right)\left(\lambda_{3}^{2}-\lambda_{2}^{2}\right)} \\
& C_{2}^{w A}=\frac{s}{2 \pi(\lambda+2 \mu) k_{a}} \frac{\left(-(\lambda+2 \mu) C_{w a}+\left(\rho_{a} k_{a} s-S_{a}\right) F_{s}\right) \lambda_{3}^{2}+\rho C_{w a} s^{2}}{\left(\lambda_{4}^{2}-\lambda_{3}^{2}\right)\left(\lambda_{2}^{2}-\lambda_{3}^{2}\right)} \\
& C_{3}^{w A}=\frac{s}{2 \pi(\lambda+2 \mu) k_{a}} \frac{\left(-(\lambda+2 \mu) C_{w a}+\left(\rho_{a} k_{a} s-S_{a}\right) F_{s}\right) \lambda_{4}^{2}+\rho C_{w a} s^{2}}{\left(\lambda_{3}^{2}-\lambda_{4}^{2}\right)\left(\lambda_{2}^{2}-\lambda_{4}^{2}\right)} \\
& C_{1}^{a S}=-\frac{\left(1-F_{s}\right)}{2 \pi(\lambda+2 \mu)\left(\lambda_{4}^{2}-\lambda_{2}^{2}\right)\left(\lambda_{3}^{2}-\lambda_{2}^{2}\right)}\left(\lambda_{2}^{2}-\frac{\left(-F_{s} C_{w a}+C_{w w}\left(1-F_{s}\right)\right)}{-k_{w}\left(1-F_{s}\right)} s\right) \\
& C_{2}^{a S}=-\frac{\left(1-F_{s}\right)}{2 \pi(\lambda+2 \mu)\left(\lambda_{4}^{2}-\lambda_{3}^{2}\right)\left(\lambda_{2}^{2}-\lambda_{3}^{2}\right)}\left(\lambda_{3}^{2}-\frac{\left(-F_{s} C_{w a}+C_{w w}\left(1-F_{s}\right)\right)}{-k_{w}\left(1-F_{s}\right)} s\right) \\
& C_{3}^{a S}=-\frac{\left(1-F_{s}\right)}{2 \pi(\lambda+2 \mu)\left(\lambda_{3}^{2}-\lambda_{4}^{2}\right)\left(\lambda_{2}^{2}-\lambda_{4}^{2}\right)}\left(\lambda_{4}^{2}-\frac{\left(-F_{s} C_{w a}+C_{w w}\left(1-F_{s}\right)\right)}{-k_{w}\left(1-F_{s}\right)} s\right) \\
& C_{1}^{a W}=\frac{s}{2 \pi(\lambda+2 \mu) k_{w}} \frac{\left(-(\lambda+2 \mu) C_{w a}+\left(\rho_{w} k_{w} s-S_{w}\right)\left(1-F_{s}\right)\right) \lambda_{2}^{2}+\rho C_{w a} s^{2}}{\left(\lambda_{4}^{2}-\lambda_{2}^{2}\right)\left(\lambda_{3}^{2}-\lambda_{2}^{2}\right)}
\end{aligned}
$$




$$
\begin{gathered}
C_{2}^{a W}=\frac{s}{2 \pi(\lambda+2 \mu) k_{w}} \frac{\left(-(\lambda+2 \mu) C_{w a}+\left(\rho_{w} k_{w} s-S_{w}\right)\left(1-F_{s}\right)\right) \lambda_{3}^{2}+\rho C_{w a} s^{2}}{\left(\lambda_{4}^{2}-\lambda_{3}^{2}\right)\left(\lambda_{2}^{2}-\lambda_{3}^{2}\right)} \\
C_{3}^{a W}=\frac{s}{2 \pi(\lambda+2 \mu) k_{w}} \frac{\left(-(\lambda+2 \mu) C_{w a}+\left(\rho_{w} k_{w} s-S_{w}\right)\left(1-F_{s}\right)\right) \lambda_{4}^{2}+\rho C_{w a} s^{2}}{\left(\lambda_{3}^{2}-\lambda_{4}^{2}\right)\left(\lambda_{2}^{2}-\lambda_{4}^{2}\right)} \\
C_{1}^{a A}=\frac{1}{2 \pi} \frac{1}{\left(\lambda_{4}^{2}-\lambda_{2}^{2}\right)\left(\lambda_{3}^{2}-\lambda_{2}^{2}\right)}\left(\lambda_{2}^{2}-K_{a}^{2}\right)\left(\lambda_{2}^{2}-\Lambda^{2}\right) \\
C_{2}^{a A}=\frac{1}{2 \pi} \frac{1}{\left(\lambda_{4}^{2}-\lambda_{3}^{2}\right)\left(\lambda_{2}^{2}-\lambda_{3}^{2}\right)}\left(\lambda_{3}^{2}-K_{a}^{2}\right)\left(\lambda_{3}^{2}-\Lambda^{2}\right) \\
C_{3}^{a A}=\frac{1}{2 \pi} \frac{1}{\left(\lambda_{3}^{2}-\lambda_{4}^{2}\right)\left(\lambda_{2}^{2}-\lambda_{4}^{2}\right)}\left(\lambda_{4}^{2}-K_{a}^{2}\right)\left(\lambda_{4}^{2}-\Lambda^{2}\right)
\end{gathered}
$$




\section{References}

[1] Abramowitz M. and Stegun I.A., Handbook of Mathematical Functions, National Bureau of Standards: Washington, DC, 1965.

[2] Arfken, G. B. and Weber, H. J., Mathematical methods for physicists, Harcourt Science and Technology Company, London, 2001.

[3] Biot M.A., General theory of the three dimensional consolidation, J. Appl. Phys. 12 (1941) 155-164.

[4] Biot M.A., Theory of propagation of elastic waves in a fluid saturated porous solid, J. Acoust. Soc. America. 28 (1956) 169-191.

[5] Bonnet G., Basic singular solutions for a poroelastic medium in the dynamic range, J. Acoust. Soc. America. 82 (1987) 1758-1762.

[6] Burridge R. and Vargas, C.A., The fundamental solution in dynamic poroelasticity, Geophys. J. Roy. Astr. S. 58 (1979) 61-90.

[7] Carcione J.M., Cavallini F., Santos J.E., Ravazzoli C.L., and Gauzellino P.M., Wave propagation in partially saturated porous media: simulation of a second slow wave, Wave Motion, 39(3) (2004) 227-240.

[8] Chen J., Time domain fundamental solution to Biot's complete equations of poroelasticity: Part I two-dimensional solution, Int. J. Sol. Struct. 31(10) (1994a) 14471490.

[9] Chen J., Time domain fundamental solution to Biot's complete equations of poroelasticity: Part II three-dimensional solution, Int. J. Sol. Struct. 31(2) (1994a) 169202.

[10] Coussy O., Mechanics of porous continua, John Wiley \& Sons, 1995.

[11] Deresiewicz H., Effect of boundaries on waves in a thermoelastic solid: Reflection of plane waves from a plane boundary, J. Mech. Phys. Solids. 8(3) (1960) 164-172. 
[12] Dominguez J., An integral formulation for dynamic poroelasticity, J. Appl. Mech. 58(2) (1991) 588-591.

[13] Dominguez J., Boundary element approach for dynamic poroelastic problems, Int. J. Numer. Methods. Eng. 35(2) (1992) 307-324.

[14] Gatmiri B., Analysis of fully Coupled Behaviour of Unsaturated Porous Medium under Stress, Suction and Temperature Gradient, Final report of CERMES-EDF, 1997.

[15] Gatmiri B., Delage P., Cerrolaza M., UDAM: A powerful finite element software for the analysis of unsaturated porous media, Adv. Eng. Software. 29(1) (1998) 29-43.

[16] Gatmiri B., Kamalian M., On the fundamental solution of dynamic poroelastic boundary integral equations in time domain, Int. J. Geomech. 2(4) (2002) 381-398.

[17] Gatmiri B., Jabbari E., Two-dimensional time-independent Green functions for unsaturated soils, In: Proceeding of 5th International Conference on Boundary Element Techniques, Lisbon, 2004a, pp. 217-221.

[18] Gatmiri B., Jabbari E., Three-dimensional time-independent Green functions for unsaturated soils, In: Proceeding of 5th International Conference on Boundary Element Techniques, Lisbon, 2004b, pp. 223-227.

[19] Gatmiri B., Jabbari E., Time-domain Green functions for unsaturated soils. Part I: Two-dimensional solution, Int. J. Sol. Struct. 42 (2005a) 5971-5990.

[20] Gatmiri B., Jabbari E., Time-domain Green functions for unsaturated soils. Part II: Three-dimensional solution, Int. J. Sol. Struct. 42 (2005b) 5991-6002.

[21] Gatmiri B., Nguyen K.V., Time 2D fundamental solution for saturated porous media with incompressible fluid, Commun. Numer. Methods Eng. 21(3) (2005) 119-132.

[22] Gatmiri B., Hoor A., Effect of excavation on the thermo-hydro-mechanical behaviour of a geological barrier, Phys. Chem. Earth 32 (2007) 947-956. 
[23] Gatmiri B., Arson C., $\theta$-Stock, a powerful tool for thermohydromechanical behaviour and damage modelling of unsaturated porous media, Comput. Geotech. 35 (2008) 890915.

[24] Gatmiri B., Maghoul P., Duhamel D., Two-dimensional transient fundamental solutions of multiphase porous media in frequency and time domain, Int. J. Sol. Struct., in press.

[25] Gatmiri B. and Eslami H., Scattering of Harmonic Waves by a Circular Cavity in a Porous Medium: Complex Functions Theory Approach, Int. J. Geomech. 7(5) (2007) 371381.

[26] Gatmiri B. and Jenab-Vossoughi B., Effects of heat convection and phase changes on heat and fluid transfer in unsaturated porous media, Heat Transfer 2000, Madrid, 2000.

[27] Gens A., Alonso E.E. and Delage, P., Computer Modeling and Applications to Unsaturated Soils, Unsaturated Soil Engineering Practice, Geotechnical Special Publication No. 68 (ed. Houston, S.L. and Fredlund, D.G.), Reston: ASCE, 1997,pp. 299330.

[28] Hemmati S., Gatmiri B., Cui Y.J., Vincent M., Implementation of a tree roots water uptake model in a finite element code $\theta$-stock, 17th International conference on soil mechanics and geotechnical engineering, Egypt, 2009.

[29] Hörmander L., Linear Partial Differential Operators, Springer: Berlin, 1963.

[30] Jabbari E., Gatmiri B., Thermo-Poro-Elastostatic Green Functions for Unsaturated Soils, Comput. Model. Eng. Sci. 18(1) (2007) 31-43.

[31] Kamalian M., Gatmiri B., Sohrabi-Bidar A. and Khalaj A., Amplification Pattern of 2D Semi-Sine Shaped Valleys Subjected to Vertically Propagating Incident Waves, Commun. Numer. Meth. Eng. 23 (2007) 871-887. 
[32] Kamalian M., Gatmiri B., Sharahi M.J., Time domain 3D fundamental solutions for saturated poroelastic media with incompressible constituents, Commun. Numer. Methods Eng., 24(9) (2008) 749-759.

[33] Kaynia, A. M. and Banerjee, P. K., Fundamental solutions of Biot's equations of dynamic poroelasticity, Int. J. Eng. Sci., 31(5) (1993) 817-830.

[34] Kupradze V.D., Gegelia T.G., Basheleishvili M.O., Burchuladze T.V., Threedimensional Problems of the Mathematical Theory of Elasticity and Thermoelasticity, North-Holland, Netherlands, 1979.

[35] Lubich C., Convolution quadrature and discretized operational calculus, I. Numerische Mathematik 52 (1988a) 129-145.

[36] Lubich C., Convolution quadrature and discretized operational calculus, II. Numerische Mathematik, 52 (1988b) 413-425.

[37] Lysmer J. and Kuhlemeyer R.L., Finite dynamic model for infinite media, J. Eng. Mech. ASCE, 95 (1969) 859-877.

[38] Maghoul P., Gatmiri B., Duhamel D., Three-dimensional Transient thermo-hydromechanical of unsaturated soils, Int. J. Numer. Anal. Methods Geomech. 2009.

[39] Manolis G.D. and Beskos D.E., Integral Formulation and Fundamental Solutions of Dynamic Poroelasticity and Thermoelasticity, Acta Mech. 76 (1989) 89-104.

[40] Norris A.N. Radiation from a point source and scattering theory in a fluid-saturated porous solid, J. Acoust. Soc. Am. 77(6) (1985) 2012-2023.

[41] Prevost J.H., Mechanics of continuous porous media. Int. J. Eng. Science 18 (1980) 787-800.

[42] Prevost J.H., Nonlinear transient phenomena in saturated porous media, Comp. Meth. Appl. Mech. Eng. 20 (1982) 3-18. 
[43] Ravichandran N., Fully coupled finite element model for dynamics of partially saturated soils, Int. J. Soil Dyn. Earthquake Eng. 29 (2009) 1294-1304.

[44] Ravichandran N. and Muraleetharan K.K. Dynamic behavior of unsaturated soils: The full formulation and its finite element solution, Proceedings, 3rd Biot Conference on Poromechanics, Norman, Oklahoma, U.S.A., 2005, pp. 489-495.

[45] Seyrafian S., Gatmiri B., Noorzad A., Green functions for a continuously nonhomogeneous saturated media, Comput. Model. Eng. Sci. 15(2) (2006) 115-126.

[46] Schanz M., Application of 3D time domain boundary element formulation to wave propagation in poroelastic solids, Eng. Anal. Boundary Elem. 25 (2001a) 363-376.

[47] Schanz M., Wave Propagation in Viscoelastic and Poroelastic Continua: A Boundary Element Approach, vol. 2 of Lecture Notes in Applied Mechanics. Berlin: Springer; $2001 b$.

[48] Schanz M. and Pryl D., Dynamic fundamental solutions for compressible and incompressible modelled poroelastic continua, Int. J. Sol. Struct. 41(15) (2004), 40474073.

[49] Stakgold I., Green's functions and boundary value problems, Pure and Applied Mathematics. John Wiley \& Sons, 2nd edition, New York, 1998.

[50] Villar M.V. and Martin P.L., Suction controlled oedometric tests in montmorillonite clay, Proc. 29th Annual Conference of the Engineering Group of the Geological Society of London, 1993, pp. 337-342.

[51] Weibe T. H. and Antes, H., A time domain integral formulation of dynamic poroelasticity, Acta Mech. 90 (1991) 125-137.

[52] White W., Valliappan, S., Lee, I.K., Unified Boundary for Finite Dynamic Models, J. of Eng. Mech. Div. ASCE. 103 (1977) 949-963. 
[53] Zienkiewicz O.C and Shiomi T., Dynamic behaviour of saturated porous media, the generalized Biot formulation and its numerical solution, Int. J. Numer. Analy. Methods. Geomech. 8 (1984) 71-96. 